\title{
Article \\ Genome-Wide Identification, Structure Characterization, Expression Pattern Profiling, and Substrate Specificity of the Metal Tolerance Protein Family in Canavalia rosea (Sw.) DC
}

\author{
Tao Zou ${ }^{1,2,3}$, Ruoyi Lin ${ }^{1,3,4}$, Lin Pu 1,3,4, Qiming Mei 1,2,4,5, Zhengfeng Wang 1,4,5,6, Shuguang Jian 1,4 and \\ Mei Zhang ${ }^{1,2, *(D)}$
}

Citation: Zou, T.; Lin, R.; Pu, L.; Mei, Q.; Wang, Z.; Jian, S.; Zhang, M. Genome-Wide Identification, Structure Characterization, Expression Pattern Profiling, and Substrate Specificity of the Metal Tolerance Protein Family in Canavalia rosea (Sw.) DC. Plants 2021, 10, 1340. https://doi.org/10.3390/ plants10071340

Academic Editor:

Jagna Chmielowska-Bąk

Received: 31 May 2021

Accepted: 29 June 2021

Published: 30 June 2021

Publisher's Note: MDPI stays neutral with regard to jurisdictional claims in published maps and institutional affiliations.

Copyright: (c) 2021 by the authors. Licensee MDPI, Basel, Switzerland. This article is an open access article distributed under the terms and conditions of the Creative Commons Attribution (CC BY) license (https:// creativecommons.org/licenses/by/ $4.0 /)$.
1 Key Laboratory of South China Agricultural Plant Molecular Analysis and Genetic Improvement \& Guangdong Provincial Key Laboratory of Applied Botany, South China Botanical Garden, Chinese Academy of Sciences, Guangzhou 510650, China; zoutao@scbg.ac.cn (T.Z.); linry@scbg.ac.cn (R.L.); pulin@scbg.ac.cn (L.P.); qmei4597@scbg.ac.cn (Q.M.); wzf@scbg.ac.cn (Z.W.); jiansg@scbg.ac.cn (S.J.)

2 Center of Economic Botany, Core Botanical Gardens, Chinese Academy of Sciences, Guangzhou 510650, China

3 University of the Chinese Academy of Sciences, Beijing 100039, China

4 CAS Engineering Laboratory for Vegetation Ecosystem Restoration on Islands and Coastal Zones, South China Botanical Garden, Chinese Academy of Sciences, Guangzhou 510650, China

5 Center for Plant Ecology, Core Botanical Gardens, Key Laboratory of Vegetation Restoration and Management of Degraded Ecosystems, Chinese Academy of Sciences, Guangzhou 510650, China

6 Southern Marine Science and Engineering Guangdong Laboratory (Guangzhou), Guangzhou 511458, China

* Correspondence: zhangmei@scbg.ac.cn

\begin{abstract}
Plant metal tolerance proteins (MTPs) play key roles in heavy metal absorption and homeostasis in plants. By using genome-wide and phylogenetic approaches, the origin and diversification of MTPs from Canavalia rosea (Sw.) DC. was explored. Canavalia rosea (bay bean) is an extremophile halophyte with strong adaptability to seawater and drought and thereby shows specific metal tolerance with a potential phytoremediation ability. However, MTP genes in leguminous plants remain poorly understood. In our study, a total of $12 \mathrm{MTP}$ genes were identified in C. rosea. Multiple sequence alignments showed that all CrMTP proteins possessed the conserved transmembrane domains (TM1 to TM6) and could be classified into three subfamilies: Zn-CDFs (five members), $\mathrm{Fe} / \mathrm{Zn}$-CDFs (five members), and Mn-CDFs (two members). Promoter cis-acting element analyses revealed that a distinct number and composition of heavy metal regulated elements and other stressresponsive elements existed in different promoter regions of CrMTPs. Analysis of transcriptome data revealed organ-specific expression of CrMTP genes and the involvement of this family in heavy metal stress responses and adaptation of $C$. rosea to extreme coral reef environments. Furthermore, the metal-specific activity of several functionally unknown CrMTPs was investigated in yeast. These results will contribute to uncovering the potential functions and molecular mechanisms of heavy metal absorption, translocation, and accumulation in C. rosea plants.
\end{abstract}

Keywords: metal tolerance protein; metal specificity; ecological adaptation; Canavalia rosea (Sw.) DC

\section{Introduction}

Heavy metals (HMs) play essential roles as the necessary cofactors of some metabolismrelated enzymes, transcription factors, and signal transduction pathways at low levels. In contrast, when they are present at high concentrations, these HMs can cause harmful effects on plant growth and cellular metabolism. Essential HMs include copper $(\mathrm{Cu})$, zinc $(\mathrm{Zn})$, manganese (Mn), nickel (Ni), and cobalt (Co). Other nonessential HMs, such as cadmium $(\mathrm{Cd})$, mercury $(\mathrm{Hg})$, silver $(\mathrm{Ag})$, and lead $(\mathrm{Pb})$, even at an extremely low level, are highly toxic to plants [1]. Basically, plants need essential HMs and acquire them from the environment, including soil, water, and air, but because their absorption process is not perfect, plants may also take up nonessential HMs or over-absorb essential HMs [2]. The toxic 
effects of nonessential HMs or excess essential HMs to plants include causing oxidative damage and metabolic disorders, resulting in chlorosis, necrosis, and growth inhibition [3].

Plants have developed a complex network of metal uptake, efflux, chelation, trafficking, and storage mechanisms to ensure precise metal homeostasis. Cation diffusion facilitator transporters (CDF, TC 2. A.4) are designated as metal tolerance proteins (MTPs) in plants and have been identified as one of the most important HM transporter families $[4,5]$. The CDF transporters were first identified by Nies and Silver [6], and numerous studies have indicated that this family exists widely in all kingdoms of life and can be classified into three subfamilies: $\mathrm{Zn}-\mathrm{CDF}, \mathrm{Fe} / \mathrm{Zn}-\mathrm{CDF}$, and Mn-CDF, mainly based on their substrate specificity [5]. Plant MTPs were further classified into seven groups according to their amino acid sequence homologies [7]. Corresponding to the former classification, the Zn-CDF subfamily comprises groups 1,5 , and 12 ; the Fe/Zn-CDF subfamily comprises groups 6 and 7, and the Mn-CDF subfamily comprises groups 8 and 9. In terms of structural features, most of the MTPs contain four to six transmembrane domains (TMDs) with cytoplasmic $\mathrm{N}$ and $\mathrm{C}$ termini, a modified signature, and a C-terminal cation efflux domain (PF01545) [5].

Until now, many studies have revealed that plant MTPs play vital roles in the absorption and detoxification of HMs, promoting their accumulation in plants [4]. The first plant MTP member was designated ZAT ( $\mathrm{Zn}$ transporter of Arabidopsis thaliana), which is an Arabidopsis orthologue of animal $Z n T$ genes (Zn transporters) [8]. ZAT was later renamed AtMTP1, and its mutation in Arabidopsis resulted in plant sensitivity to $\mathrm{Zn}$, while overexpression of AtMTP1 showed a tolerant phenotype to $\mathrm{Zn}$ in transgenic Arabidopsis [9]. As an important divalent metal transporter, the substrate and metal specificity of different MTP members generated interest in both nutrient metal uptake and phytoremediation of HM contamination [10-15]. Arabidopsis contains 12 MTP genes. In addition to AtMTP1, AtMTP3 has been proven to be involved in $\mathrm{Zn}$ and cobalt (Co) tolerance [16], and AtMTP11 is a manganese $(\mathrm{Mn})$ transporter $[17,18]$. AtMTP12 can interact with AtMTP5t1 and transport $\mathrm{Zn}$ into the Golgi [19]. AtMTP8 transports both $\mathrm{Mn}$ and iron (Fe) [20-22]. Rice is a cadmium (Cd)-concentrating crop, and OsMTP1 shows a certain Cd-transporting ability, mediates $\mathrm{Cd}$ absorption in plants, and transports Zn and $\mathrm{Ni}$ [3,23]. OsMTP1 overexpression in tobacco also showed enhanced $\mathrm{Cd}$ accumulation and tolerance, implying that it could be used in phytoremediation [12]. Both OsMTP8 and OsMTP11 can transport Mn across a transmembrane and are involved in Mn tolerance in rice [24-30]. In addition, nonhyperaccumulator cucumber MTP members showed $\mathrm{Zn}$ transporting features and regulatable subcellular localization patterns [31].

Notably, in some metal hyperaccumulators, MTP members seem to contribute more greatly to metal tolerance and enrichment in plants. Thlaspi goesingense can hyperaccumulate $\mathrm{Ni} / \mathrm{Zn}$ in vivo, and TgMTP1 shows a high level of expression in T. goesingense and functions to enhance $\mathrm{Ni}, \mathrm{Zn}, \mathrm{Co}$, and $\mathrm{Cd}$ tolerance when overexpressed in yeast $[32,33]$. In transgenic Arabidopsis, TgMTP1 is likely to act in transporting $\mathrm{Zn}$ into the vacuole, therefore enhancing both $\mathrm{Zn}$ accumulation and tolerance [34]. Arabidopsis halleri is a natural Zn hyperaccumulator. AhMTP1 can transport Zn, and compared to its homolog AtMTP1 in Arabidopsis, AhMTP1 shows a much higher expression level in A. halleri than AtMTP1 in Arabidopsis [35]. Further research showed that gene expansion has occurred in the AhMTP1 locus in the A. halleri genome [36]. Furthermore, other plant MTPs isolated from metal hyperaccumulators, such as ShMTP1 from Stylosanthes hamata (Mn tolerant) [37], PtdMTP1 from hybrid poplar (Zn/Ni tolerant) [10], SaMTP1 from Sedum alfredii Hance (Zn tolerant) [38], and BjCET2/3/4 from Brassica juncea (Zn/Cd tolerant) [39,40], showed typical HM transporting or accumulation features.

As a mangrove-associated and halophyte species distributed widely in tropical and subtropical seashore and arid coral reefs, Canavalia rosea (Sw.) DC. exhibits tenacious vitality under high salinity, extreme drought, and even some HM-polluted coastal areas. It has been proposed that halophytes can also enrich HMs and that their ability to tolerate HM stress relies on similar physiological and molecular mechanisms to those exhibited 
under high saline-alkaline stress [41]. Halophyte can cope with several abiotic constraints occurring simultaneously in their natural environment by sequestering absorbed toxic ions (including salty ions and HMs) in trichomes or vacuoles, as well as maintaining regular biomass production and relatively stable plant water status for plant growth. These characteristics cause some halophytes to be promising candidates for phytoremediation management of heavy-metal-polluted areas in saline environments [42]. Some saline shrubs or mangrove species have been proven to possess the abilities to absorb and accumulate salt and toxic metals from saline soils efficiently and therefore can be used as potential candidate engineering plants for the restoration of saline soils contaminated with heavy metals [43-47]. Some reports have also proven that halophytes adopt similar physiological, biochemical, and molecular mechanisms to cope with the toxic levels of salinity and heavy metals $[41,42,48]$. In China and many other countries, coastal wetlands show severe HM pollution due to emissions from inland rivers [49]. Mangrove species can be used for ecological restoration and phytoremediation of vegetation in tropical and subtropical coral sand and coastal beaches because of their strong tolerance for salt and HM mixed pollution. Therefore, it is necessary to determine the molecular mechanisms of this special habitat's halophyte, C. rosea, for adsorption and enrichment of HM from polluted water and soil. In addition to many genome sequences for plant species, characterization of gene families has also been concerned with gene expression and biochemical functional identification, especially from evolutionary biology. Based on our previous genomic sequence project on C. rosea, the CrMTP sequences were isolated from its genome data, and a total of 12 CrMTPs were selected for further research. In this study, $C r M T P s^{\prime}$ evolution and structural features were systematically analyzed, as well as the promoters' characteristics and gene expression patterns. RNA-seq experiments were also performed to identify the possible biological functions of this gene family responding to HMs or other abiotic stressors. Moreover, their potential metal substrates were also explored by a yeast heterologous expression assay with a series of mutant strains. This study provides a basis for the systematic understanding of the C. rosea MTP family involved in HM translocation, offering potential scientific evidence for halophytes applying for phytoremediation purposes, and further illuminates the roles of plant MTPs involved in adaptation to extreme adversity.

\section{Results}

\subsection{An Overview of C. Rosea CrMTP Genes}

A total of 12 CrMTP genes were identified from the C. rosea genome. Bioinformatics analysis showed that all CrMTPs contained the Cation_efflux domain (PF01545), thereby suggesting that these possess a basic characteristic of the MTP family. Among them, seven CrMTPs possessed a ZT_dimer domain (PF16916) near their C-terminals, which often lie within the cytoplasm and form homodimers during protein activities. Based on their sequence similarity to Arabidopsis MTPs and the gene nomenclature system, the $C$. rosea MTPs were correspondingly designated CrMTP1 to CrMTP12 (Table 1). The length of the CrMTP coding DNA sequences ranged from $1032 \mathrm{bp}$ (CrMTP1) to $2592 \mathrm{bp}$ (CrMTP12) with 343-863 amino acid residues. Members of the CrMTP gene family were subdivided into classes $\mathrm{Zn}-\mathrm{CDF}, \mathrm{Mn}-\mathrm{CDF}$, and $\mathrm{Zn} / \mathrm{Fe}-\mathrm{CDF}$ according to their homologies with AtMTPs (Figure 1).

The physicochemical parameters, including the molecular weight (MW), theoretical isoelectric point (pI), and grand average of hydropathicity (GRAVY) values of the CrMTP proteins were predicted in Table 1. The MWs ranged from $40.758 \mathrm{kD}$ (CrMTP5) to $97.802 \mathrm{kD}$ (CrMTP12), and most of them were within 40-50 kD. Their theoretical pI values, between 5.08 and 7.77, showed that CrMTPs are basically neutral (Table 1). The GRAVY results, ranging from -0.137 (CrMTP10) to 0.146 (CrMTP4), indicated that the CrMTPs generally had weak hydrophilicity, which was consistent with the MTPs' biochemical functions as transmembrane transporters (Table 1). Transmembrane helices (TMHs) predication using TMHMM and PHYRE $^{2}$ was almost consistent with little differences (Figure S1A,B), and the topologies, including the embedded directions of transmembrane 
helices in CrMTPs, indicated the functional diversity of different CrMTP members diffusing the metals (Table 1 and Figure S1C).

Table 1. Identified CrMTPs of Canavalia rosea (Sw.) DC. and their molecular characteristics.

\begin{tabular}{|c|c|c|c|c|c|c|c|}
\hline \multirow{2}{*}{ Gene } & \multirow{2}{*}{$\begin{array}{l}\text { Protein } \\
\text { Length }\end{array}$} & \multirow{2}{*}{$\begin{array}{c}\text { MW } \\
(\mathbf{k D a})\end{array}$} & \multirow{2}{*}{ PI } & \multirow{2}{*}{ GRAVY } & \multirow{2}{*}{ TMHs\&Topologies * } & \multicolumn{2}{|c|}{ Subcellular Localization } \\
\hline & & & & & & Plant-mPLoc & WoLF_PSORT \\
\hline CrMTP1 & 433 & 48.182 & 6.19 & 0.034 & $6 /$ in to in & Vacuole & $\begin{array}{l}\text { plas: } 7 \text {, E.R.: } 4 \text {, cyto: } 1 \\
\text { mito: } 1 \text {, vacu: } 1\end{array}$ \\
\hline CrMTP4 & 396 & 43.767 & 6.18 & 0.146 & $6 /$ in to in & Vacuole & $\begin{array}{l}\text { plas: 5, vacu: 4, E.R.: 4, } \\
\text { golg: } 1\end{array}$ \\
\hline CrMTP5 & 363 & 40.758 & 7.31 & 0.071 & $5 /$ in to out & Vacuole & $\begin{array}{l}\text { plas: } 6 \text {, nucl: } 2 \text {, E.R.: } 2 \text {, } \\
\text { chlo: } 1 \text {, cyto: } 1 \text {, vacu: } 1 \text {, } \\
\text { golg: } 1\end{array}$ \\
\hline CrMTP6 & 614 & 67.268 & 7.77 & -0.084 & $2 /$ out to out & Vacuole & $\begin{array}{l}\text { chlo: } 4 \text {, mito: } 4 \text {, nucl: } 2 \text {, } \\
\text { golg: } 2 \text {, cyto: } 1 \text {, plas: } 1\end{array}$ \\
\hline CrMTP7 & 431 & 47.853 & 7.32 & -0.013 & $4 /$ in to in & Vacuole & $\begin{array}{l}\text { plas: } 5.5 \text {, E.R.: } 4 \text {, cyto_plas: } \\
\text { 3.5, mito: } 2 \text {, chlo: } 1 \text {, pero: } 1\end{array}$ \\
\hline CrMTP8 & 506 & 56.719 & 6.11 & -0.013 & $4 /$ out to out & $\begin{array}{l}\text { Mitochondrion; } \\
\text { Vacuole }\end{array}$ & $\begin{array}{l}\text { chlo: } 6 \text {, mito: } 2, \text { E.R.: } 2, \\
\text { nucl: } 1 \text {, cyto: } 1 \text {, plas: } 1 \text {, } \\
\text { extr: } 1\end{array}$ \\
\hline CrMTP8.1 & 409 & 46.004 & 5.25 & -0.010 & $4 /$ in to in & Vacuole & $\begin{array}{c}\text { plas: } 7.5, \text { cyto_plas: } 4.5, \\
\text { vacu: } 3 \text {, E.R.: } 3\end{array}$ \\
\hline CrMTP9 & 412 & 46.950 & 6.84 & -0.119 & $3 /$ out to in & Vacuole & plas: 8, E.R.: 5, golg: 1 \\
\hline CrMTP9.1 & 396 & 45.485 & 7.32 & -0.072 & $5 /$ in to out & $\begin{array}{l}\text { Cell membrane; } \\
\text { Vacuole }\end{array}$ & $\begin{array}{c}\text { plas: } 10 \text {, vacu: } 2 \text {, cyto: } 1 \text {, } \\
\text { E.R.: } 1\end{array}$ \\
\hline CrMTP10 & 398 & 45.777 & 6.48 & -0.137 & $5 /$ in to out & $\begin{array}{l}\text { Cell membrane; } \\
\text { Vacuole }\end{array}$ & plas: 12, vacu: 2 \\
\hline CrMTP11 & 396 & 45.080 & 5.08 & 0.008 & $4 /$ out to out & $\begin{array}{l}\text { Cell membrane; } \\
\text { Vacuole }\end{array}$ & plas: 12 , vacu: 2 \\
\hline CrMTP12 & 863 & 97.802 & 7.16 & -0.079 & $13 /$ out to in & Vacuole & plas: 12 , chlo: 1 , pero: 1 \\
\hline
\end{tabular}

* Here, the TMHs\&Topologies analyses uses the prediction results generated from TMHMM Server V. 2.0 (http://www.cbs.dtu.dk/ services/TMHMM/, (accessed on 1 May 2021)).

Subcellular localization prediction for CrMTPs was performed with different programs (Table 1). The results indicated that CrMTPs were widely distributed in different endomembrane parts of organelles, as well as in cell plasma membranes. Generally speaking, as a kind of membrane protein, the presence of transmembrane domains in each CrMTP member provides necessary conditions for cation cross-membrane transportation (Figure S1), while the detailed subcellular localization status of specific CrMTP might be determined more by protein modification, protein interaction, or even metal binding, which was presumed as a dynamic and regulated process and also as a manner for metal distribution in vivo when under excess HM stress.

The physical map of the CrMTP family showed an uneven distribution of CrMTP genes on the 11 chromosomes of $C$. rosea - the most genes on chromosome 2 (three genes), followed by chromosomes 3 and 8 with two genes each, whereas chromosomes 1,5, and 7 carried no CrMTP genes (Figure 2). Synonymous (Ks) and nonsynonymous (Ka) values were calculated to explore the selective pressures on the duplication of $\mathrm{CrMTPs}$ based on all nucleotide sequences. The results revealed that two gene pairs, CrMTP8/CrMTP8.1 and CrMTP9/CrMTP9.1, possessed Ka/Ks ratios greater than 0.1 that were lower than 1 , indicating that they underwent some purifying selection and demonstrated segmental duplications, which might suggest their evolution and functional divergence (Table 2).

Table 2. Ka/Ks analysis and duplicated type calculation for CrMTP genes.

\begin{tabular}{cccccc}
\hline Duplicated Pair & Duplicate Type & Ka & Ks & Ka/Ks & Positive Selection \\
\hline CrMTP8/CrMTP8.1 & Segmental & 0.0961 & 0.4000 & 0.2403 & No \\
CrMTP9/CrMTP9.1 & Segmental & 0.0610 & 0.606 & 0.1006 & No \\
\hline
\end{tabular}




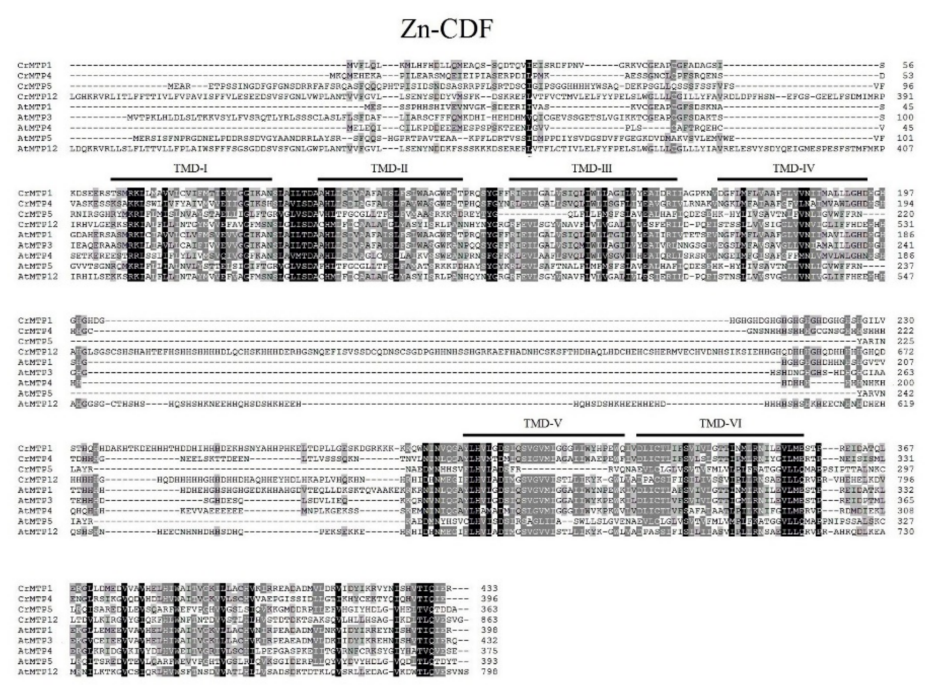

(A)

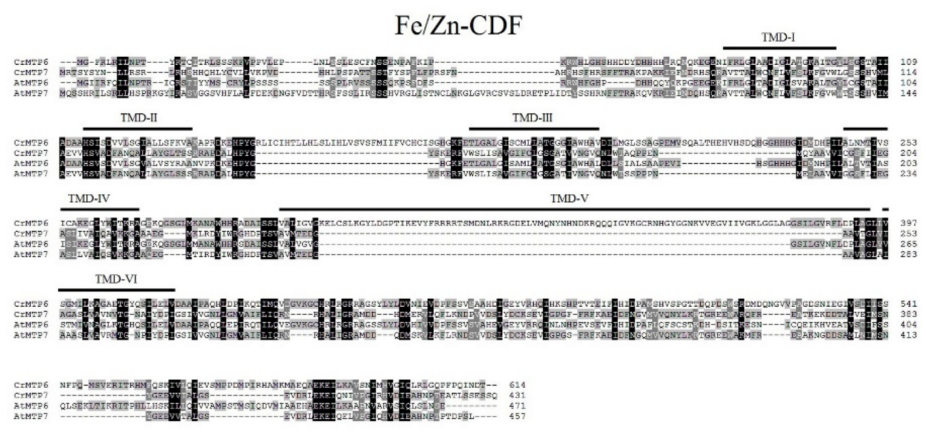

(B)

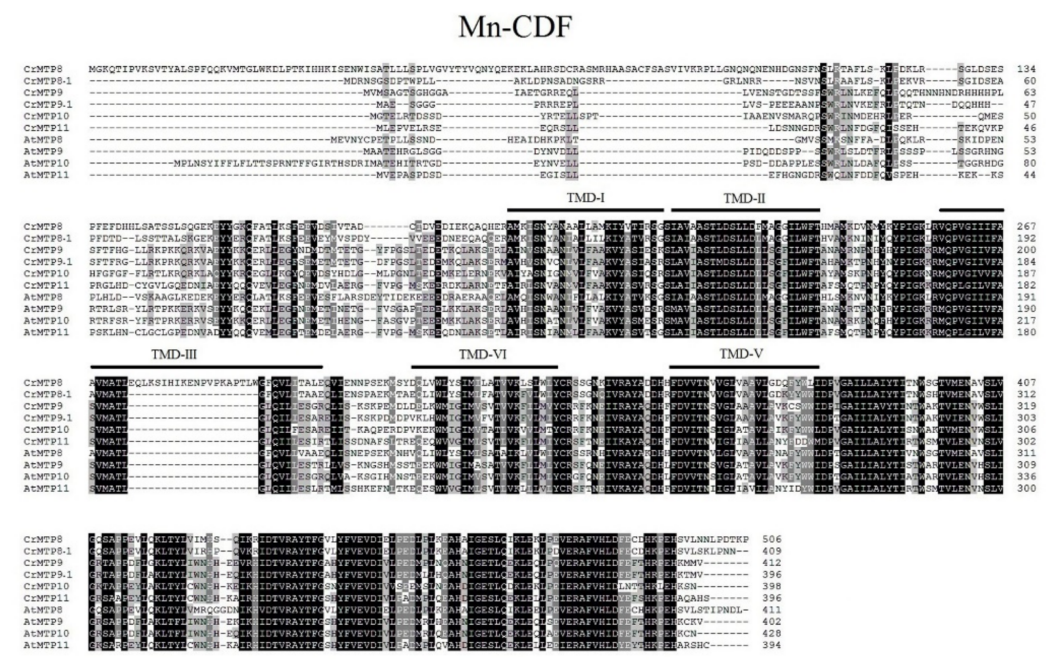

(C)

Figure 1. Multiple sequence alignment of CrMTP and AtMTP proteins. A total of 12 CrMTP proteins were identified in Canavalia rosea (Sw.) DC. and AtMTP proteins from Arabidopsis thaliana were classified into Zn-CDFs (A), Fe/Zn-CDFs (B), and Mn-CDFs (C) and then aligned using ClustalW. The transmembrane domains (TMDs I-VI) were marked with bold lines. 


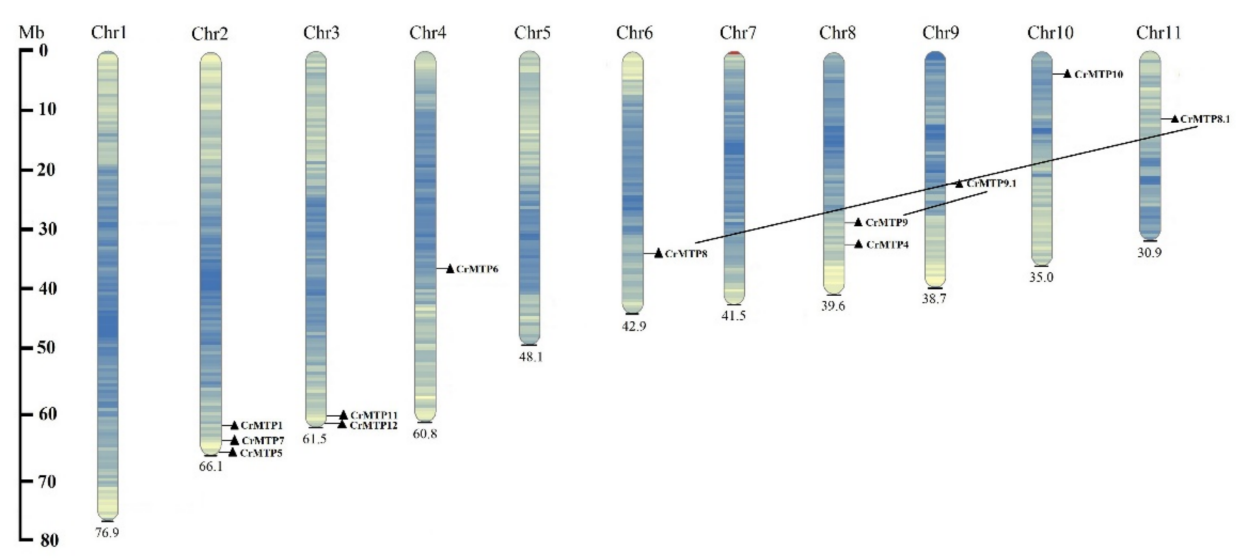

Figure 2. Chromosomal location and gene duplication of 12 CrMTP genes in the C. rosea genome.

\subsection{Multiple Sequence Alignment and Phylogenetic Analysis of the CrMTP Family}

CrMTP protein sequences were further aligned with AtMTPs according to a previous report (Figure 1) [14]. The results indicated that the MTPs were highly conserved in phylogeny even in different plant species. As shown in Figure 1, 12 CrMTP proteins were classified into three major subgroups, namely, Zn-CDF, Fe/Zn-CDF, and Mn-CDF. The transmembrane domains (TMDs) of the CrMTPs predicted by TMHMM that were critical for cation channel establishment are marked in Figure 1. These domains displayed higher conservation than other sequences, while the N-terminus of all CrMTPs showed great variation, which might be related to specific post-translational modifications, protein interactions, or subcellular localization. In the $\mathrm{Zn}-\mathrm{CDFs}$ subgroup, most of the members in this subgroup had a variable histidine-rich (His-rich) loop between TMD-IV and TMD-V, which has been proven to be critical to metal selectivity or as sensors of the cellular metal concentration in plant MTPs $[15,50]$. The His-rich loops of Zn-CDFs from Arabidopsis and C. rosea varied greatly in length and histidine content (Figure 1A), further indicating the fine regulation mechanisms of MTPs caused by metal substrates. The Zn/Fe-CDF subgroup showed the greatest variability both in TMDs and other sequences, and more specifically, the CrMTP6 showed an extra sequence (KELCSLKGYLDGPTIKEVYFRRRRTSMDNLRKRGDELVMQNYNHNDKRQQQIGVKGCRNHGYGGNKVVEGVIIVGKLGGLAG) in the putative TMD-V (Figure 1B), which was quite different from other MTP6s [14] and might destroy the topological structure of CrMTP6 and affect the biological function for transporting metals. In addition, Mn-CrCDF subgroups, including CrMTP8, CrMTP8.1, CrMTP9, CrMTP9.1, CrMTP10, and CrMTP11, were more conserved than the other two subgroups (Figure 1C).

To study the evolutionary relationships between $C$. rosea MTP proteins and known MTPs from Arabidopsis, soybean, rice, and poplar, an unrooted neighbor-joining phylogenetic tree was created based on multiple alignments of the predicted sequences of the MTP proteins from these plants. There were no AtMTP2 or AtMTP 3 orthologous genes found in the $C$. rosea genome, similar to those found in the soybean genome (Figure 3 ), while the Mn-CDF subgroup members, CrMTP8, CrMTP8.1, CrMTP9, and CrMTP9.1, seemed to have doubled compared with other plant species [14], which is in agreement with previous reports [51-53]. This indicates gene duplication events. Ka versus Ks substitutions $(\mathrm{Ka} / \mathrm{Ks})$ were estimated with nucleotide sequences of CrMTP8/8.1 and CrMTP9/9.1 gene pairs, which showed segmental duplication events within the $C$. rosea genome as paralogs (Table 2). 


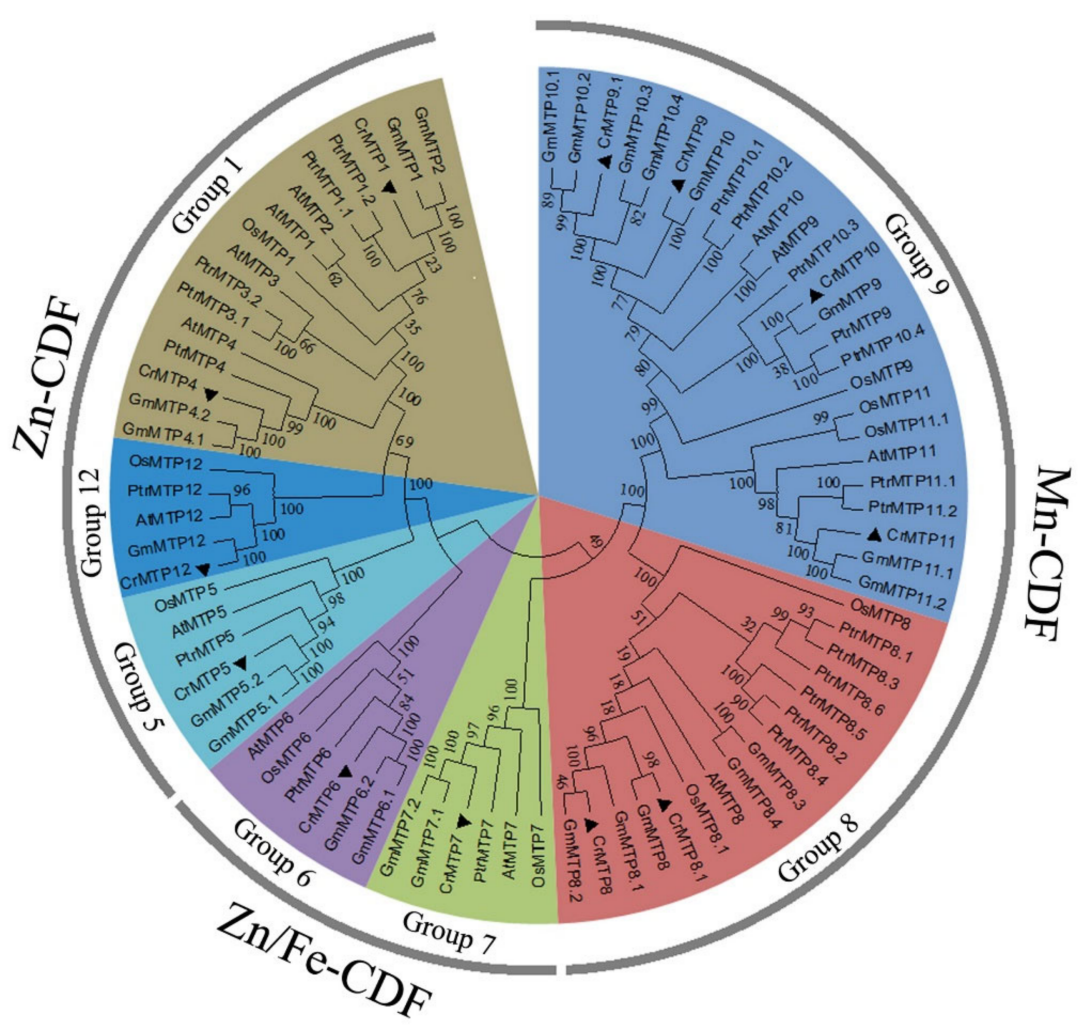

Figure 3. Phylogenetic tree of CrMTPs from C. rosea, Arabidopsis, soybean, rice, and poplar. The neighbor-joining (NJ) tree was constructed using Clustal W and MEGA 6.0 software with 1000 bootstrap replicates.

\subsection{Gene Structure and Conserved Motifs of CrMTP Members}

The gene exon-intron organization for each CrMTP was examined to analyze the evolution of this gene family further. As shown in Figure 4, in the coding region sequences of CrMTP members, Zn-CDF members, CrMTP1, CrMTP4, and CrMTP12 were intronless, while other CrMTP members contained 5-12 introns. CrMTP genes that clustered closely showed similar exon numbers and gene structures (Figure 4), which was consistent with the results of phylogenetic analysis and classification of MTP families in other species $[14,51,52,54]$. Notably, the gene structure of different $M T P$ families also represented a certain degree of similarity and conservation in different plant species.
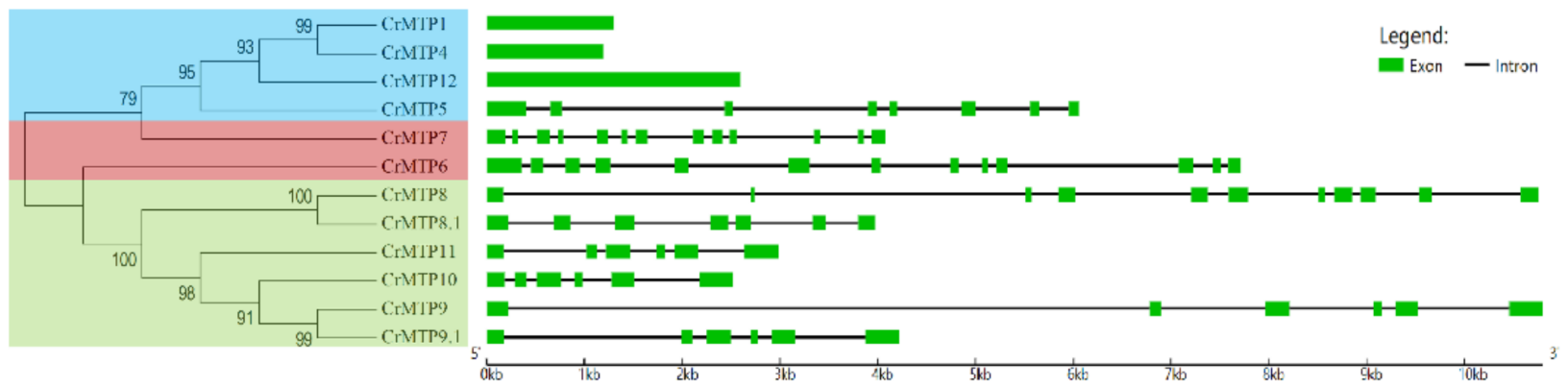

Figure 4. CrMTPs' classification and gene structures of CrMTP genes in C. rosea. The left part shows that the CrMTPs phylogenetic tree is classified into three subgroups ( $\mathrm{Zn}-\mathrm{CrCDFs}$, in blue; Fe/Zn-CrCDFs, in red; Mn-CrCDFs, in green). The left part shows the exon-intron gene structures of all CrMTPs.

To understand the possible biochemical roles and substrate specificities of $C$. rosea MTP proteins, the conserved CDF domain (PF01545) and ZT_dimer domain (PF16916) 
were investigated (Figure 5A). Protein domain analyses supported that all CrMTP proteins possessed the typically conserved functional CDF domain, which contained signature sequences of CDF members (Figure 1) [5]. Half of the CrMTP members (CrMTP6 and five Mn-CDF-type CrMTPs) had the ZT dimer, which belongs to an important zinc transporter dimerization domain (Figure 5A). The conserved motifs search also indicated that the members of the same cluster or group in the CrMTP family had similar motif types and distributions (Figure 5B).

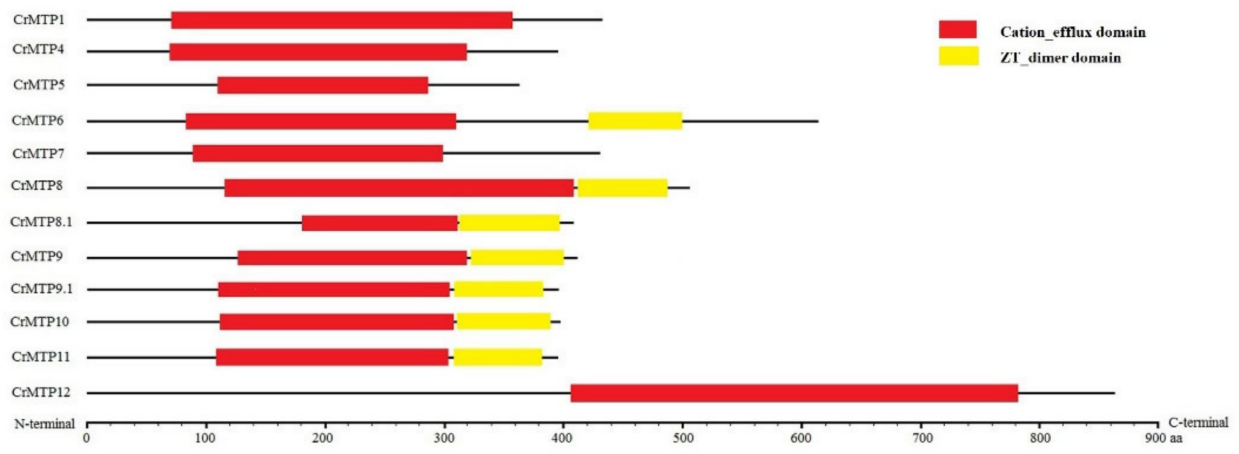

(A)

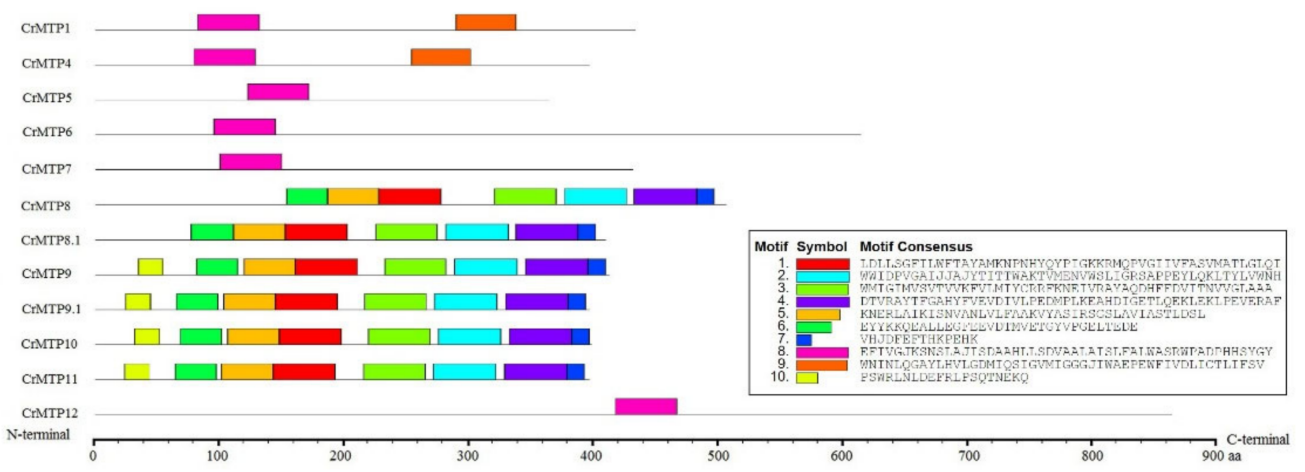

(B)

Figure 5. Conserved domains searched by the Pfam database and their conserved motifs discovered by Multiple Em for Motif Elicitation (MEME) motif discovery server of CrMTP proteins in C. rosea. (A) The protein families (PFAM) domain diagrams of CrMTPs, searched by PFAM database; (B) Conserved motifs of CrMTPs, identified using Multiple EM for Motif Elicitation (MEME) database. The websites of PFAM and MEME databases were listed in "Materials and Methods" part.

\subsection{Heavy-Metal-and Abiotic-Stress-Related cis-Acting Elements in CrMTP Promoters}

To obtain further insight into the putative biological function of CrMTP genes in C. rosea, cis-acting elements located in the promoter and untranslated regions (UTR) of each gene were investigated. The regulation of CrMTP expression remains a key mediator of biological functions, especially in response to HM stresses. In this study, putative cis-acting elements were identified in the promoter regions of all CrMTPs by scanning the CDS upstream 2000 bp sequences of CrMTP members with the online PlantCARE program.

The cis-acting regulatory elements in the CrMTP promoter regions searched online were simply classified into 11 categories: light-responsive elements, gibberellinresponsive elements, MeJA-responsiveness elements, auxin-responsive elements, salicylic acid-responsiveness elements, ABA-responsive elements (ABRE), ethylene-responsive elements (ERE), MYC-binding elements (MYC), MYB binding site involved in droughtinducibility (MBS), and TC-rich repeats (cis-acting element involved in defense and stress responsiveness). In summary, the numbers of these elements in each CrMTP promoter region were specific (Figure 6A), indicating that the expression of each CrMTP gene could be systematically regulated by a series of environmental or developmental factors. In 
addition, because MTPs play an important role in maintaining plant metal tolerance and homeostasis, three kinds of metal-responsive elements (MRE1, MRE2, and CuRE) were manually searched. These HM stress-related cis-acting elements were summarized within $12 \mathrm{CrMTP}$ promoter regions (Figure $6 \mathrm{~B}$ ), and the results showed that $\mathrm{CuRE}$ was the most dominant and was present in almost all CrMTP promoter regions (except CrMTP11). MRE1 existed in CrMTP7 and CrMTP10 promoter regions, while MRE2 existed in CrMTP4 and CrMTP8.1 promoter regions. Further functional studies are still necessary to confirm the functions of these cis-acting elements in the regulation of CrMTPs' expression patterns.

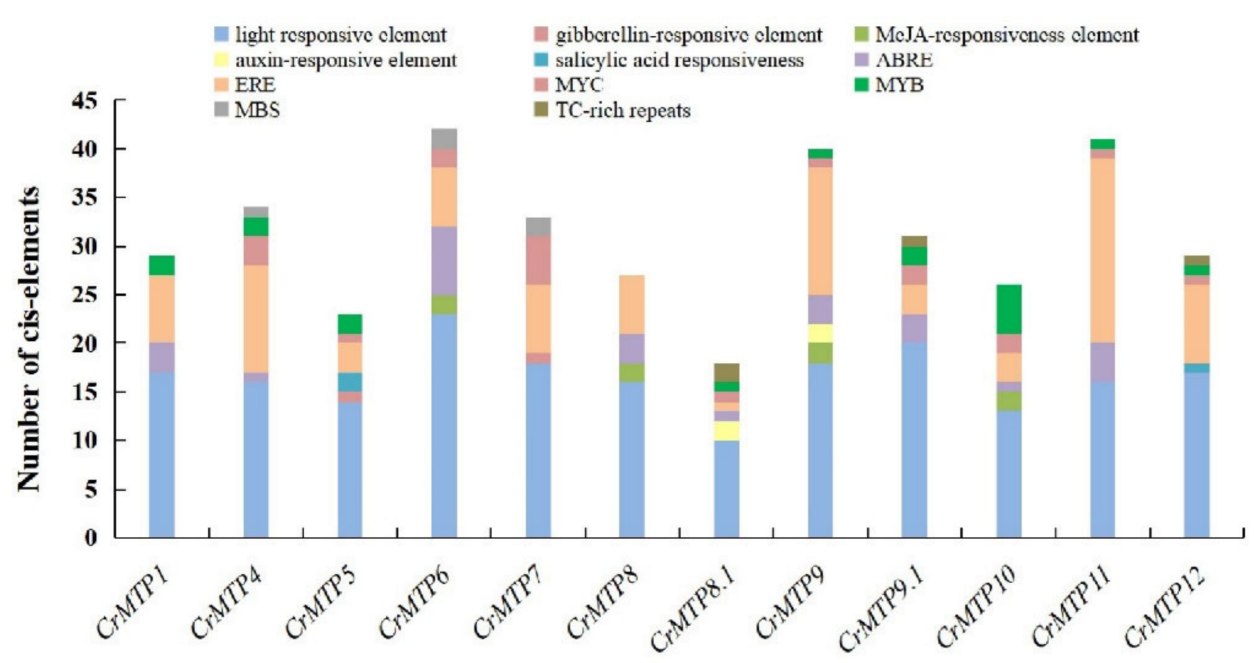

(A)
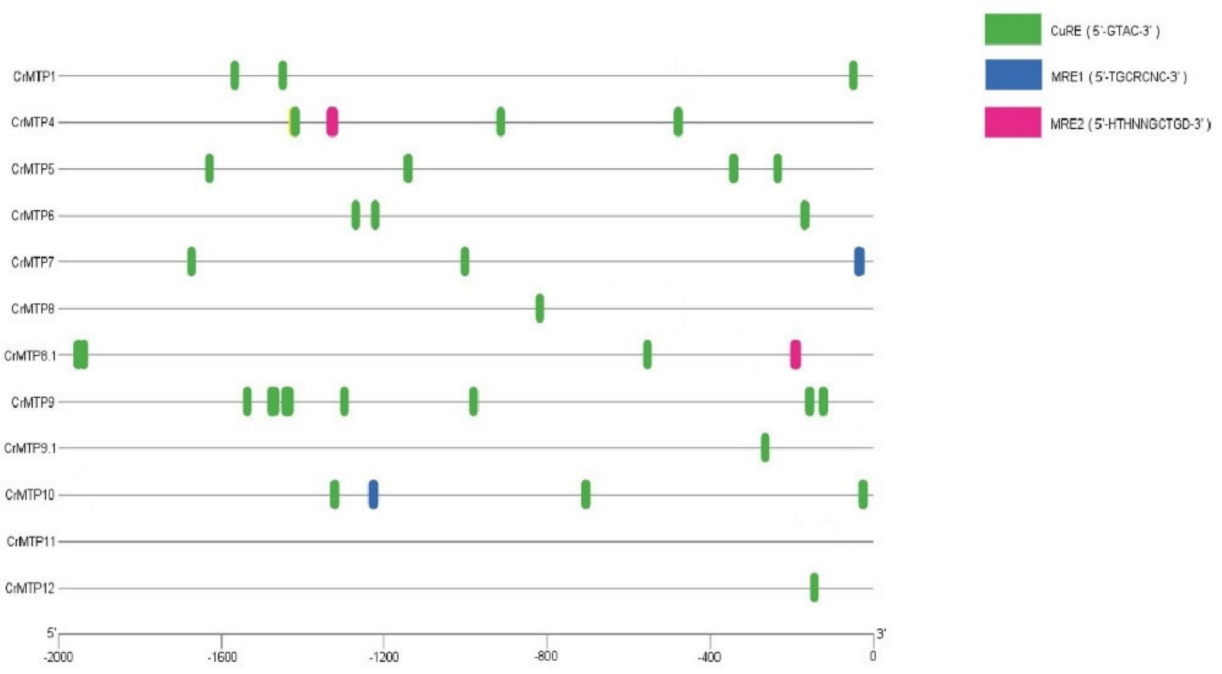

(B)

Figure 6. Predicted cis-acting elements in CrMTP promoter regions of $C$. rosea. The $2000 \mathrm{bp}$ sequence upstream of 12 CrMTP genes' coding region was analyzed by PlantCARE and manually searched with MRE1, MRE2, and CuRE: (A) different cis-acting elements were identified and plotted against a bar diagram. The abundance of different regulatory elements on each of the promoters is shown in different colors; (B) the location of metal stress responding cis-acting elements (MRE1, MRE2, and CuRE) in 12 CrMTP promoter regions.

\subsection{Expression Profiles of CrMTPs in Different Tissues or under Different Stress Treatments}

To investigate whether the predicted CrMTP genes were actually transcribed, their transcription levels were examined in different tissues using RNA-seq. As shown in 
Figure 7, eight genes (CrMTP1, 4, 5, 6, 7, 8, 8.1, and 12) were highly expressed in all five tissues. The expression levels of the other members (CrMTP9, 9.1, 10, and 11) varied among these tissues.
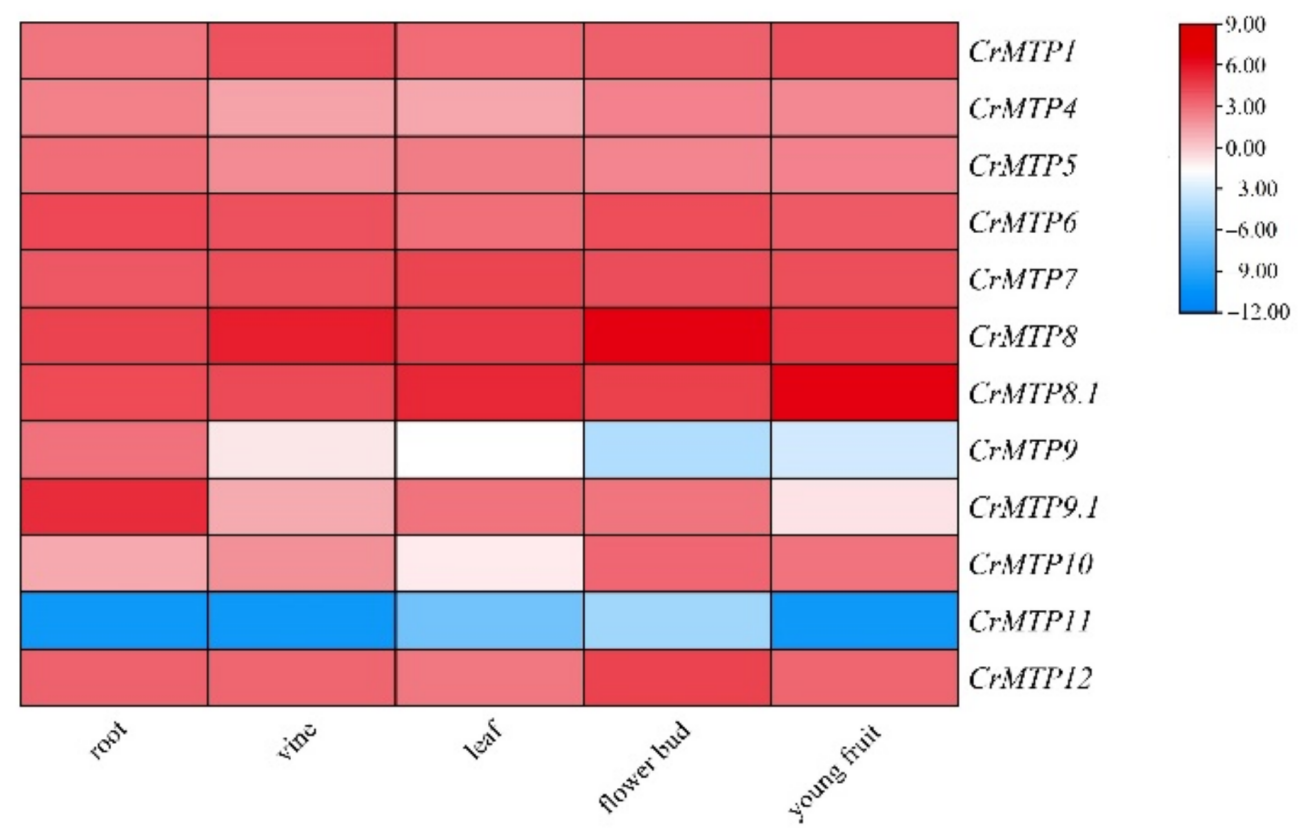

Figure 7. Heatmaps showing the expression patterns of CrMTPs in various tissues (root, vine, leaf, flower bud, and young fruit) of $C$. rosea plants. The expression level of each gene is shown with the values of FPKM ( $\log 2)$. Red denotes high expression levels, and blue denotes low expression levels.

To explore the biological functions of CrMTP genes further, expression changes in the entire MTP family of $C$. rosea seedlings were analyzed in the presence of different metal ions, including $\mathrm{Zn}, \mathrm{Cd}, \mathrm{Mn}$, and $\mathrm{Cu}$. The CrMTPs showed various responses to the same metal ion either in the roots or leaves (Figure 8), and the expression of certain genes slightly changed under different metal treatments (Figure 8 and Figure S2). In root samples, except CrMTP8 and CrMTP10, the other 10 CrMTPs showed slightly downregulated expression levels under different $\mathrm{HM}$ challenges, either at 2 or $48 \mathrm{~h}$ (Figure 8A). However, overall, the expression levels of CrMTP genes remained relatively stable in leaves, and only CrMTP1 and CrMTP9.1 showed slightly elevated expression changes under different $\mathrm{HM}$ challenges, both at 2 and $48 \mathrm{~h}$ (Figure 8B). When comparing two control samples (CK $2 \mathrm{~h}$ and CK $48 \mathrm{~h}$ ) in root samples, CrMTP8, CrMTP9, and CrMTP10 generally showed elevated expression levels in the CK $48 \mathrm{~h}$ sample, compared to the CK $2 \mathrm{~h}$ sample. In leaf samples, only CrMTP11 showed greatly induced expression in the CK $48 \mathrm{~h}$ samples, compared to the $\mathrm{CK} 2 \mathrm{~h}$ samples. Considering that $\mathrm{C}$. rosea is a mangrove-associated species, seawater logging is normal in their growing habitats; therefore, the obvious expression changes in specific CrMTP members are possibly an adaptive mechanism for the normal growth of C. rosea plants.

To further explore the possible roles of CrMTPs in C. rosea's adaptation to special habitat with extreme adversity on tropical coral reefs, RNA-seq data for CrMTP members were also extracted from transcriptome sequencing for mature leaves captured from $\mathrm{YX}$ Island and SCBG (Figure 9). Only CrMTP1 and CrMTP12 showed slightly downregulated expression changes in the YX Island leaf sample, while in the SCBG sample, CrMTP10 and CrMTP11 showed greatly induced expression. 

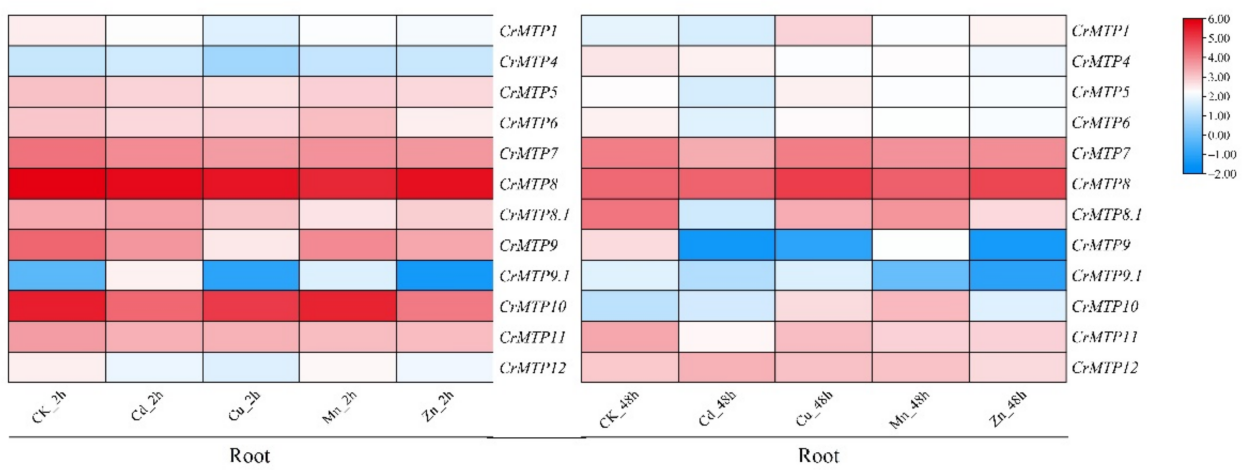

(A)
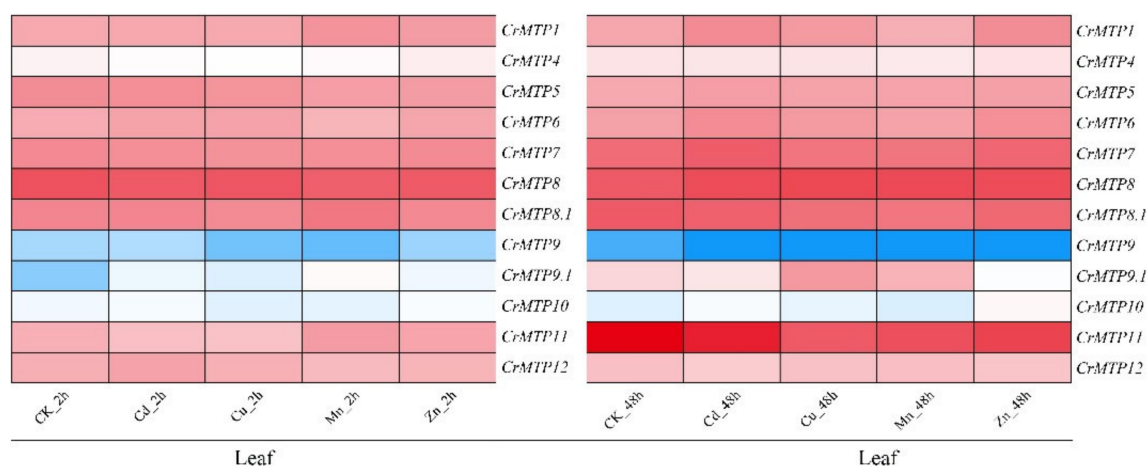

(B)

Figure 8. Heatmaps showing the expression patterns of $C r M T P s$ under different $\mathrm{HM}$ challenges $\left(0.1 \mathrm{mM} \mathrm{CdCl}_{2}, 0.1 \mathrm{mM} \mathrm{CuSO}_{4}, 1 \mathrm{mM} \mathrm{MnCl}_{2}\right.$, and $\left.0.5 \mathrm{mM} \mathrm{ZnSO}_{4}\right)$ both in roots (A) and in leaves (B). The expression level of each gene is shown with the values of FPKM (log2). Red denotes high expression levels, and blue denotes low expression levels.

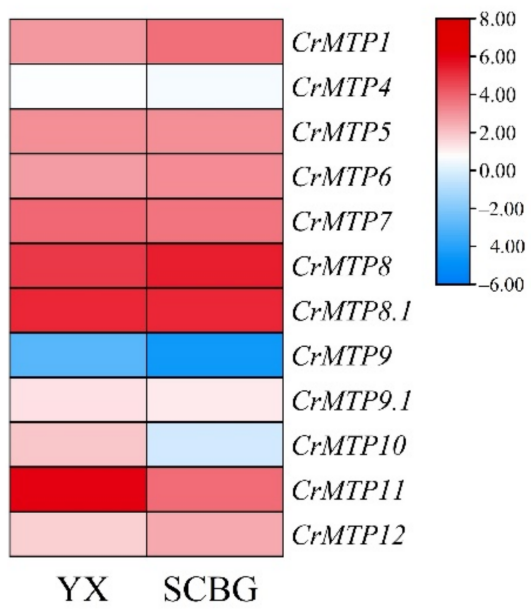

Figure 9. Heatmaps showing the expression differences of the CrMTPS in mature C. rosea leaves planted in South China Botanical Garden (SCBG) and in Yongxing Island (YX). The expression level of each gene is shown with the values of FPKM $(\log 2)$. Red denotes high expression levels, and blue denotes low expression levels.

Four-week-old C. rosea seedling plants were subjected to high salinity and ABA treatments, and the expression changes were also detected by qRT-PCR assays. The relative expression levels of CrMTPs in response to salt stress and ABA in C. rosea roots, vines, and leaves varied to different degrees (Figure S3). Generally, high salinity decreased the expression levels of most CrMTP members, and ABA upregulated some CrMTP members' 
expression in all three tested tissues of C. rosea seedlings (CrMTP1, CrMTP4, CrMTP6, and (rMTP9).

\subsection{Functional Characterization of CrMTPs in Yeast}

For functional characterization of different CrMTP members, the complete open reading frame (ORF) of the seven $C$. rosea MTPs were PCR amplified. The yield recombinant vectors for CrMTPs' heterologous expression in yeast (CrMTPs-pYES2) are shown in Figure S4. Interestingly, by using cDNA from C. rosea seedling roots or leaves as a template, five CrMTP members could not be amplified successfully. Specifically, CrMTP6 and CrMTP7 had relatively high expression in different $C$. rosea tissues (Figure 10). This was likely caused by primer specificity or more detailed expression patterns of these CrMTP members. Furthermore, the other seven CrMTP members amplified by RT-PCR showed identical sequences with bioinformatic analysis results.

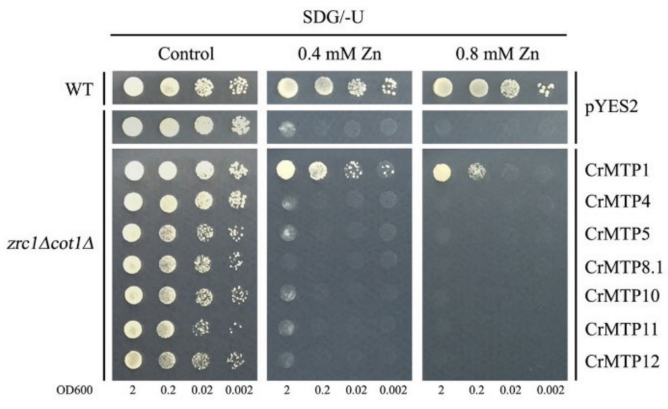

(A)

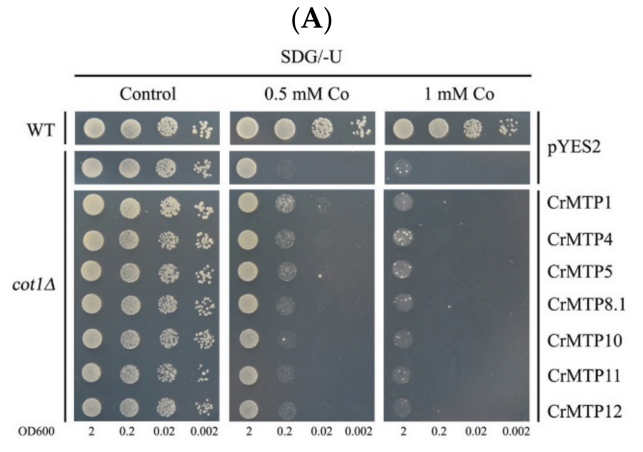

(C)

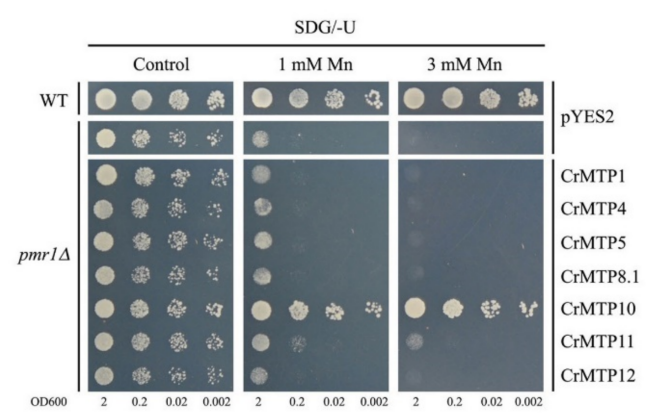

(E)

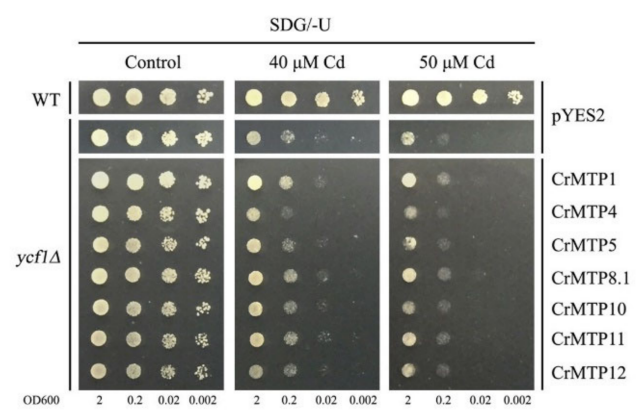

(B)

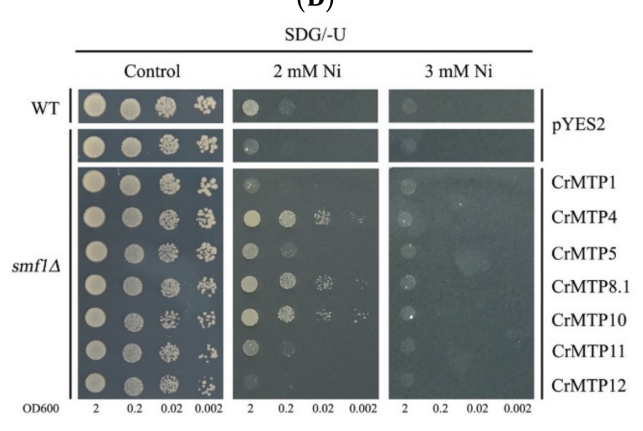

(D)

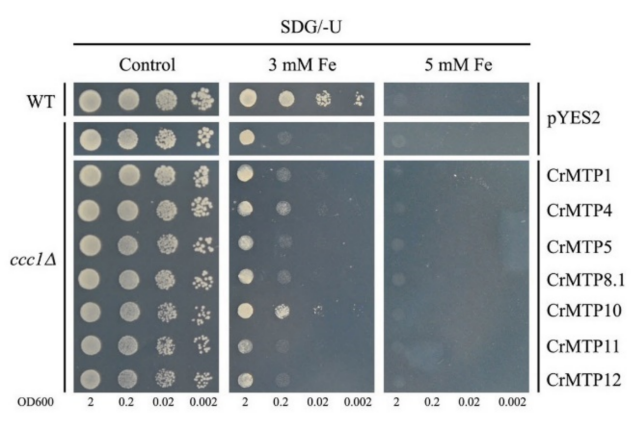

(F)

Figure 10. Complementation of yeast mutants on solid medium containing heavy metals. S. cerevisiae wild-type strain BY4741 was transformed with the empty vector PYES2, and mutant strains were transformed with the empty vector pYES2 or with the vectors carrying the CrMTP gene. Yeast cultures were adjusted to OD600 $=2$, and $2 \mu \mathrm{L}$ of serial dilutions (10-fold, from left to right in each panel) were spotted on SDG-Ura medium supplemented with 0.4 or $0.8 \mathrm{mM} \mathrm{ZnSO}_{4}(\mathbf{A}), 40$ or $50 \mu \mathrm{M} \mathrm{CdCl} 2$ (B), 0.5 or $1 \mathrm{mM} \mathrm{CoCl}_{2}(\mathbf{C}), 2$ or $3 \mathrm{mM} \mathrm{NiCl}_{2}$ (D), 1 or $3 \mathrm{mM} \mathrm{MnCl}_{2}$ (E), and 3 or $5 \mathrm{mM} \mathrm{FeCl}_{2}(\mathbf{F})$ on SDG-Ura plates. The corresponding yeast spots growing on SDG-Ura plates without heavy metals were used as controls. The plates were incubated for $2-5$ days at $30^{\circ} \mathrm{C}$. The images are representative of three independent experiments. 
After the ORFs were inserted into yeast expression vector $\mathrm{pYES2}$, the role of CrMTPs in $\mathrm{Zn}^{2+}, \mathrm{Cd}^{2+}, \mathrm{Co}^{2+}, \mathrm{Ni}^{2+}, \mathrm{Mn}^{2+}$, and $\mathrm{Fe}^{2+}$ transport was determined by testing the complementation of the yeast mutants' phenotype. The double mutant $z r c 1 \Delta \cot 1 \Delta$ was defective for both $\mathrm{Zn}^{2+}$ transporter $\mathrm{ZRC1}$ and $\mathrm{Co}^{2+}$ transporter COT1 and highly sensitive to $\mathrm{Zn}$ [15]. As shown in Figure 10A, the expression of CrMTP1 could rescue the sensitivity of $z r c 1 \Delta \cot 1 \Delta$ to $\mathrm{Zn}$, while other members could not. The complementary capability of CrMTPs to the Cd-sensitive phenotype of $z r c 1 \Delta \cot 1 \Delta$ was also tested; however, the effects were not visible (data not shown). ycf1 $\Delta$ was highly sensitive to $C d$, and none of these seven CrMTP members could completely complement the Cd sensitivity phenotype of ycf1 $\Delta$ mutant, although CrMTP1, CrMTP8.1, and CrMTP11 seemed to slightly improve the Cd tolerance (Figure 10B). Similarly, CrMTP1, CrMTP4, and CrMTP5 slightly improved the Co tolerance of $\cot 1 \Delta$ (Figure 10C). At relatively lower Ni concentration (2 mM), CrMTP4, CrMTP8.1, and CrMTP10 significantly improved the Ni tolerance of $s m f 1 \Delta$, while at higher Ni concentration (3 mM), the effects were not visible (Figure 10D). Although CrMTP8.1, CrMTP10, and CrMTP11 are all Mn-CDF members, only CrMTP10 obviously improved the Mn tolerance of $p m r 1 \Delta$, and CrMTP11 only showed a slight effect (Figure 10E). The only two Fe/Zn-CDF members CrMTP6 and CrMTP7 were not successfully cloned, while in the Fe sensitivity assay of yeast mutant $\operatorname{ccc} 1 \Delta$, both CrMTP4 and CrMTP10 enhanced yeast growth on plates with lower Fe $(3 \mathrm{mM})$ (Figure 10F). Overall, the ability to transport different bivalent cations of CrMTPs depends on both member specificity and concentration of cations.

\section{Discussion}

The cation diffusion facilitators (CDFs) family is a ubiquitous family of membranebound HM transporters involved in metal tolerance/resistance. Cation diffusion facilitators are found in all major phyla of living organisms, including Archaea, Eubacteria, and Eukaryotes $[7,55]$. Plant CDFs are commonly called metal tolerance proteins (MTPs). To date, the MTP gene family has been reported in a number of plant species, but information on this family is limited in leguminous plants. In this study, the C. rosea genome was comprehensively searched for MTP family analysis, which resulted in the identification of 12 putative CrMTP genes.

Plant MTP family members belong to divalent cation $\left(\mathrm{Zn}^{2+}, \mathrm{Co}^{2+}, \mathrm{Fe}^{2+}, \mathrm{Cd}^{2+}, \mathrm{Ni}^{2+}\right.$, and $\mathrm{Mn}^{2+}$ ) transporters, which are mainly involved in metal ion efflux from the cytoplasm, either to the outside of the cell or into subcellular compartments [5]. In plants, MTPs usually form three clusters (Zn-CDF, Fe/Zn-CDF, and Mn-CDF) according to their substrate specificity or seven groups $(1,5,6,7,8,9$, and 12) according to the results of the phylogenetic analysis and annotation of Arabidopsis MTPs [7]. In the present study, 12 CrMTP genes were successfully identified from the $C$. rosea genome, which was similar to that of other Arabidopsis (12) and rice (10) [7]. Compared with MTP family identification of other plant species, only two gene pairs (CrMTP8/8.1, CrMTP9/9.1) showed gene expansion, and different subfamilies of Zn-CDFs (five), Fe/Zn-CDFs (five), and Mn-CDFs (two) were reasonable. Thus far, along with whole-genome sequencing in plants, the MTP gene families have been systematically identified in several plant species. For example, the typical gene expansion of the PtrMTP family was obvious in Populus trichocarpa, which contained 22 PtrMTP members [51]. The MTP family in Brassica rapa var. rapa (turnip) was more expanded than that in Arabidopsis, including 18 BrrMTP members [54]. N. tabacum contained at least 26 NtaMTPs, which were mostly due to its allotetraploid feature. Correspondingly, in N. sylvestris and N. tomentosiformis, there were only 13 NsyMTPS and 12 NtoMTPs that could be detected in their diploid genomes [52]. Similarly, there were at least 20 TaMTP sequences in modern wheat Triticum aestivum (hexaploid, AABBDD) [56]. However, in sweet orange (Citrus sinensis) and grape (Vitis vinifera) genomes, there were 12 and 11 members of CitMTPs and VvMTPs, respectively [14,57]. Additionally, there were 13 CsMTPs identified from Mn hyperaccumulator tea plants (Camellia sinensis) [53]. Canavalia rosea is a typical diploid species with 11 pairs of chromosomes and 12 CrMTP 
members in total. Our findings in this study suggest that the C. rosea MTP family is relatively conserved in terms of the number and type of members.

As a mangrove-associated species, $C$. rosea holds great potential for HM storage and recycling, similar to other halophyte or mangrove species [41,46,58]. Exploring the functional characteristics of the metal ion transporters in C. rosea is of great interest. Canavalia rosea is a seashore halophytic plant with excellent extreme saline-alkaline resistance and can be used as an ecological restoration pioneer species, both on artificial islands and reefs for vegetation construction and on estuaries and littoral zones for HM phytoremediation in tropical and subtropical regions. After $C r M T P$ genes' identification, the expression patterns of 12 CrMTP genes of C. rosea were analyzed (Figure 7, Figure 8, Figure 9, Figure 10). The results demonstrated variation in the transcriptional levels among different CrMTP clusters. In general, three Zn-CDF members (CrMTP1, CrMTP4, CrMTP5, and CrMTP12) and two $\mathrm{Fe} / \mathrm{Zn}$-CDF members (CrMTP6 and CrMTP7) in C. rosea showed almost constitutively high expression in all tested tissue types, and only two Mn-CDF members (CrMTP8 and CrMTP8.1) also had a similarly high expression level (Figure 7). Highly expressed genes usually play important roles in plant development and stress responses. Our results indicated that these eight $C r M T P$ s might be important regulatory factors for $C$. rosea plant growth. In contrast, the other four Mn-CDF members (CrMTP9, CrMTP9.1, CrMTP10, and CrMTP11) were expressed at obviously different levels in the five tested tissue types of C. rosea, implying that these CrMTP genes could be transcriptionally regulated by growth and developmental specific factors and that they might have specific functions in those corresponding tissues. The CrMTP expression patterns generally varied slightly under different HM treatments both in leaves and roots (Figure 8), which was quite different from previous reports $[38,53,54]$, in which some MTPs were obviously upregulated following exposure to excessive amounts of some HMs. In some plant species, the identified MTP members showed relatively stable expression patterns even under multiple HM challenges, and only a small minority of HM stressors could affect the expression of MTPs [51,52]. In this study, most CrMTPs showed relatively higher expression levels in seedling leaves than in roots (Figure 8), indicating that the HM diffusion mediated by MTPs in aerial parts is more necessary and important than that in underground parts when under HM challenge. Conversely, as CrMTPs are dynamically regulated endomembrane transporters for cations, the changeable expression in roots seems to be more effective for controlling the absorption or diffusion of excess HMs.

Cis-acting elements are a series of nucleotide motifs that bind to specific transcription factors (TFs), thereby regulating transcription in plants [59]. The expression pattern of genes mostly depends on the way that TFs bind to the promoter regions. Here, the numbers and categories of cis-acting elements in CrMTPs' promoter regions were systematically analyzed and summarized. Most of the promoter regions of CrMTPs contained some specific cis-acting elements, such as ABRE, MYC, MYB, MBS, and TC-rich repeats, which are involved in binding some specific TFs or responding to some biotic or abiotic stressors. Three are also some hormone-related responsive elements, suggesting that the CrMTPs could be regulated or affected by different stressors (Figure 6). Three kinds of metalresponsive elements (MRE1, MRE2, and CuRE) were also manually analyzed in detail. The promoter regions of CrMTPs showed more variability than the gene coding region, which corresponded with their gene-specific expression pattern (Figure 5B, Figure 6). The contribution of single CrMTPs to HMs or other stress tolerances and environmental adaptations in C. rosea needs to be explored further.

Canavalia rosea is highly adaptive to tropical coastal areas and therefore can be used as pioneer species for ecological restoration of the intertidal zones and coral islands or reefs. Although there is limited experimental evidence that plant MTPs are involved in high salinity and drought tolerance, some previous reports indicated that MTPs might be related to some adaptations to abiotic stressors, except HMs. The expression of Arabidopsis MTPs was determined under various abiotic stressors in both root and shoot tissues by RNA-seq, and AtMTP9 showed the highest level of expression in response to salt and high 
osmotic stress [57]. A cation diffusion facilitator gene from soybean, GmCDF1 (GmMTP12), negatively regulates salt tolerance by regulating $\mathrm{K}^{+}-\mathrm{Na}^{+}$homeostasis [60]. A novel CDF (MceT) identified from a moderate halophile, Planococcus dechangensis, evolved from a traditional $\mathrm{Zn}^{2+}$ diffusion facilitator and acted as a $\mathrm{Na}^{+}\left(\mathrm{Li}^{+}, \mathrm{K}^{+}\right) / \mathrm{H}^{+}$antiporter [61]. In our study, further habitat-specific RNA-seq data indicated that five CrMTP members could be transcriptionally regulated by special habitats with high salinity and drought characteristics (YX sample) (Figure 9), which might be an adaptive consequence for $C$. rosea growing and breeding in tropical coral islands or reefs.

The roles of MTPs have been extensively researched previously in many other plant species. These proteins are mainly involved in the transmembrane transport of divalent metal ions, mainly including ion absorption and efflux, cellular distribution, and other stress response. The first identified plant MTP was AtMTP1, which was demonstrated to be a transition metal transporter that extrudes metal ions from the cytoplasm to vacuolar regions, playing pivotal roles in maintaining zinc homeostasis in Arabidopsis [62]. Subsequently, several MTP members were identified from metal hyperaccumulator plants Stylosanthes hamata, Arabidopsis halleri, and Thlaspi goesingense [33,35,37]. These MTPs have certain features in common, that is, their relatively high expression in vivo is positively associated with their metal accumulating characteristic. Another experimental system with yeast as a host for a heterologous expression assay also confirmed this conclusion $[23,28,33]$. Here, the metal substrate specificity of CrMTPs was detected with a series of yeast mutant strains. Our results indicated that CrMTP1 is a typical Zn transporter with a slight capability for $\mathrm{Cd}$ and Co transport (Figure 10A-C). Similar findings were also found in rice OsMTP1 [3,23], and as a consequence, OsMTP1 showed great Cd hyperaccumulating activity in tobacco and was endowed with promising potential for phytoremediation [12]. Although CrMTP4, CrMTP5, and CrMTP12 were classified as Zn-CDF, they did not show $\mathrm{Zn}$ transport in yeast, which was consistent with previous studies [51,52]. Although two Fe-CDFs (CrMTP6 and CrMTP7) were not cloned successfully, CrMTP10 showed typical Fe transporting capability in yeast (Figure 10F). Three Mn-CDFs (CrMTP8.1, CrMTP10, and CrMTP11) were cloned, while only CrMTP10 showed a Mn-transporting capability in yeast (Figure 10E). CrMTP4, CrMTP5, CrMTP8.1, CrMTP10, and CrMTP11 showed various degrees of a Ni-transporting capability, indicating that these members possessed metal substrate specificity (Figure 10D). This may be due to their unique amino acid composition in their His-loops (CrMTP4 and CrMTP5) or other loops and transmembrane domains. In general, this protein family might fulfill an important function in HM tolerance and regulate the environmental adaptation of $C$. rosea to tropical coastal regions, although more research is needed for further clarification.

\section{Materials and Methods}

\subsection{Plant Materials and Stress Treatments}

Canavalia rosea plants growing in the South China Botanical Garden (SCBG, $23^{\circ} 18^{\prime} 76^{\prime \prime} \mathrm{N}$, $113^{\circ} 37^{\prime} 02^{\prime \prime} \mathrm{E}$ ) and leaf samples taken from C. rosea plants growing on Yongxing Island (YX, $16^{\circ} 83^{\prime} 93^{\prime \prime} \mathrm{N}, 112^{\circ} 34^{\prime} 00^{\prime \prime} \mathrm{E}$ ) were used in this study. The seeds of $\mathrm{C}$. rosea were gathered from coastal regions of Hainan Province, China, and then cultivated under normal conditions (22-26 ${ }^{\circ} \mathrm{C}$ with a photoperiod of $16 \mathrm{~h}$ light $/ 8 \mathrm{~h}$ darkness, and 50-60\% relative air humidity) in a growth chamber, in which the cultured plants were kept in artificially controlled, optimum growing conditions. The seeds were germinated and cultivated in a soil/vermiculite mixture for one month before exposure to the various heavy metal (HM) or other stressors for further transcriptional analyses of CrMTP members. In brief, $C$. rosea seedlings with similar growth vigor and status were used in the following treatments. The seedlings were removed from the pots and carefully washed with distilled water to remove soil from the roots and transferred into different treatment solutions (1/2 Hoagland solution). For HM stress treatments, $C$. rosea seedlings were subjected to $0.1 \mathrm{mM} \mathrm{CdCl}_{2}, 0.5 \mathrm{mM} \mathrm{ZnSO}_{4}, 1 \mathrm{mM} \mathrm{MnCl}_{2}$, and $0.1 \mathrm{mM} \mathrm{CuSO}_{4}$ with the roots submerged. For high salinity and ABA treatment, seedlings were soaked in $600 \mathrm{mM} \mathrm{NaCl}$ solution (1/2 Hoagland solution). For ABA treatment, the C. rosea seedling leaves were sprayed with a freshly prepared working solution of $100 \mu \mathrm{M}$ exogenous ABA. 
Plant tissues were collected at different time points ( $2 \mathrm{~h}$ and $48 \mathrm{~h}$ for RNA-seq, and $2 \mathrm{~h}$ and $24 \mathrm{~h}$ for qRT-PCR). All samples were immediately frozen in liquid nitrogen and stored at $-80{ }^{\circ} \mathrm{C}$ for subsequent gene expression analysis. Three independent biological replicates were used.

\subsection{Identification, $\mathrm{Ka} / \mathrm{Ks}$ Calculation, and Evolutionary Analyses of the CrMTP Family in C. Rosea}

Whole-genome sequencing was performed with $C$. rosea plants growing in Hainan Province, China. The assembled genome data of $C$. rosea were annotated with different programs, including InterPro [63] and Pfam [64] databases for gene family identification and DIAMOND [65] and InterProscan [63] to acquire all C. rosea proteins' information with conserved domains and motifs $\left(\mathrm{e}<1 \times 10^{-5}\right)$. Subsequently, Pfam ID (Cation_efflux, PF01545) was used to search for CrMTP family members, and putative sequences of CrMTP proteins were identified and submitted to the NCBI Conserved Domain Database (https://www.ncbi.nlm.nih.gov/Structure/cdd/wrpsb.cgi, accessed on 1 May 2021) to confirm the presence of the Cation_efflux domain. Finally, candidate CrMTPs were named based on their sequence homology with Arabidopsis MTPs or other plant species MTPs and $C$. rosea genome annotation.

Metal tolerance protein (MTP) sequences from Arabidopsis thaliana, Oryza sativa, Glycine max, and Populus trichocarpa were obtained from the Arabidopsis Information Resource (TAIR, http: / / www.arabidopsis.org, accessed on 1 May 2021), the Rice Genome Annotation Project (RGAP, http:/ / rice.plantbiology.msu.edu/index.shtml, accessed on 1 May 2021), and Phytozome (https:/ / phytozome.jgi.doe.gov/pz/portal.html, accessed on 1 May 2021), respectively. The obtained MTP nucleotide and protein sequences from C. rosea and G. max are listed in Table S1. The protein sequences of 12 AtMTPs from Arabidopsis, 10 OsMTPs from rice, 22 PtrMTPs from P. trichocarpa, 24 GmMTPs from G. max, and 12 CrMTPs from C. rosea were used to construct a neighbor-joining (NJ) phylogenetic tree using Clustal W and MEGA 6.0 software with 1000 bootstrap replicates.

The CrMTP genomic DNA and cDNA sequences were downloaded from the C. rosea genome database. Gene segmental duplication events of the CrMTP family were analyzed using MCScanX software (http:/ / chibba.pgml.uga.edu/mcscan2/, accessed on 1 May 2021). The number of synonymous substitutions per synonymous site (Ka), the number of nonsynonymous substitutions per nonsynonymous site (Ks), and the probability (P-value) of Fisher's exact test of neutrality were calculated using the Nei-Gojobori model with 1000 bootstrap replicates [66]. The diagrams of exon/intron organization, protein structure, chromosomal location, and gene duplication events were drawn by TBtools software [67]. The exon-intron structures within the coding sequences and the genomic sequences of each CrMTP gene were predicted with the Gene Structure Display Server (GSDS, http:/ / gsds.cbi.pku.edu.cn, accessed on 1 May 2021). The conserved motifs of CrMTPs were detected using Multiple Em for Motif Elicitation (MEME) software (http: / / meme-suite.org/tools/meme, accessed on 1 May 2021), with the maximum number of motifs set at 10 .

\subsection{Protein Properties and Sequence Analyses}

The molecular weight and isoelectric points of predicted CrMTPs were detected using the ExPASy proteomics server (https: / / web.expasy.org/protparam/, accessed on 1 May 2021). The TMHMM Server 2.0 program (http:/ / www.cbs.dtu.dk/services/TMHMM/, accessed on 1 May 2021) and Protein Fold Recognition Server tool (PHYRE², http:/ / www. sbg.bio.ic.ac.uk/phyre2/html/page.cgi?id=index, accessed on 1 May 2021) were employed to predict the transmembrane helices and topologies of CrMTPs, and PHYRE ${ }^{2}$ was also used to perform the 3D prediction of CrMTPs. WoLF_PSORT and the online Plant-mPLoc server (http: / / www.csbio.sjtu.edu.cn/bioinf/plant-multi/, accessed on 1 May 2021) were used for subcellular localization prediction. 


\section{4. cis-Acting Elements Analysis of CrMTP Promoters}

The promoter regions (2000 bp upstream from the translation start site, TSS) of all CrMTPs were retrieved from the genome database of $C$. rosea. The cis-acting elements present in these regions were predicted with PlantCARE (http:/ / bioinformatics.psb.ugent. be/webtools/plantcare/html/, accessed on 1 May 2021). MRE1 (5'-TGCRCNC-3') (metalresponse element 1) [68], MRE2 (5'-HTHNNGCTGD-3') (metal-response element 2) [69], and CuRE (5'-GTAC-3') (copper response element) [70], which are related to metal stress, were also searched manually in each CrMTP promoter region. These cis-acting elements were summarized with Microsoft Excel 2010 software (Microsoft Corp., Albuquerque, NM, USA), and several selected CrMTP promoters were visualized using TBtools.

\subsection{RNA-Seq of Different C. Rosea Tissues or under Different Stress Treatments}

C. rosea RNA-seq datasets were constructed using Illumina HiSeq X sequencing technology. First, seven different tissues from C. rosea plants (root, vine, young leaf, flower bud, and young silique samples) were collected from C. rosea plants growing in the SCBG; mature leaf samples from $C$. rosea growing in SCBG and on YX Island were examined using FastQC (http:/ / www.bioinformatics.babraham.ac.uk/projects/fastqc/, accessed on 1 May 2021) based on the primary $40 \mathrm{~Gb}$ clean reads and were mapped to the $C$. rosea reference genome using Tophat v.2.0.10 (http:/ / tophat.cbcb.umd.edu/, accessed on 1 May 2021). Secondly, the $C$. rosea seedling tissues under different $\mathrm{HMs}$ and other abiotic stress challenges were also sequenced at the transcriptome level, and all EST information was mapped to the $C$. rosea reference genome. Gene expression levels were calculated as fragments per kilobase $(\mathrm{kb})$ of transcript per million mapped reads (FPKM) according to the length of the gene and the read counts mapped to the gene: FPKM = total exon fragments/[mapped reads (millions) $\times$ exon length $(\mathrm{kb})]$. Expression levels of CrMTPs were visualized as clustered heatmaps $(\log 2)$ using TBtools, which were directly shown with FPKM values by Microsoft Excel 2010.

\subsection{Quantitative RT-PCR Analysis}

Total RNA of the root, vine, and leaf samples were extracted separately using the plant RNA extraction kit (TIANGEN BIOTECH, Beijing, China) according to the manufacturer's instructions. RNA concentration and quality were tested by NanoDrop 1000 (Thermo Fisher Scientific, Waltham, MA, USA), with the integrity checked on $0.8 \%$ agarose gel. The expression levels of 12 CrMTPs were determined by quantitative reverse transcription PCR (qRT-PCR), and three biological replications for each treatment were conducted. In brief, a total of $1 \mu \mathrm{g}$ of RNA was reverse transcribed into cDNA in a $20 \mu \mathrm{L}$ reaction volume using AMV reverse transcriptase (TransGen Biotech, Beijing, China) according to the supplier's instructions. To quantify the relative transcript levels of selected CrMTP genes, qRT-PCR was performed with gene-specific primers using the LightCycler480 system (Roche, Basel, Switzerland) and TransStart Tip Green qPCR SuperMix (TransGen Biotech, Beijing, China) according to the manufacturer's instructions. The gene-specific primers used for this analysis are listed in Table S2. All gene expression data obtained via qRT-PCR were normalized to the expression of CrEF-1 $\alpha$ (Table S2).

\subsection{Functional Identification with a Yeast Expression System}

The full-length cDNA sequences of the CrMTP genes were obtained from the genome database of $C$. rosea. Then, the open reading frames (ORFs) of CrMTP genes were PCR amplified from different cDNA samples of $C$. rosea with gene-specific primer pairs (listed in Table S2). After several PCR procedures, the PCR fragments were purified and cloned into the BamHI and EcoRI sites of pYES2 to yield recombinant plasmids of pYES2-CrMTPs and sequenced. The Saccharomyces cerevisiae wild-type (WT) strain BY4741 (Y00000) and five deletion mutants ycf1 $\Delta$ (Y04069), pmr1 $\Delta$ (Y04534), cot1 $\Delta$ (Y01613), smf1 $\Delta$ (Y06272), and $\operatorname{ccc} 1 \Delta$ (Y04169) were obtained from Euroscarf (http:/ / www.euroscarf.de, accessed on 1 May 2021). The double-mutant yeast strain $z r c 1 \Delta / \cot 1 \Delta$ was kindly provided by Yuan and Sanders [15]. The plasmids were introduced into yeast using the LiAc/PEG method [71]. 
Yeast growth and metal sensitivity tests were performed as previously described, with minor modifications [23]. Briefly, transformed yeast was grown on solid SDG-Ura medium (synthetic dropout medium plus $2 \%$ galactose, uracil deficiency) plates for two days, and single colonies of yeast transformants were selected and used to inoculate liquid SDG-Ura medium. It was then incubated overnight or longer at $30^{\circ} \mathrm{C}$, diluted with fresh prewarmed SDG medium (volume ratio 1:10), and then incubated with vigorous shaking for two days at $30{ }^{\circ} \mathrm{C}$ to reach an optical density of 2 at OD600 (optical density at $600 \mathrm{~nm}$ ). The cells were then serially diluted in 10-fold steps, and $2 \mu \mathrm{L}$ aliquots of each were finally spotted onto SDG medium plates with or without metal stressors. Plates were incubated at $30{ }^{\circ} \mathrm{C}$ for two to five days and photographed.

\subsection{Statistical Analyses}

All analyses were conducted in triplicate, with the results shown as the mean $\pm \mathrm{SD}$ $(n \geq 3)$. The Excel 2010 (Microsoft Corporation, Albuquerque, NM, USA) Statistics program was used to perform statistical analyses.

\section{Conclusions}

In this study, a total of 12 CrMTP genes were identified from the C. rosea genome using bioinformatic methods. Subsequently, according to their phylogenetic relationship with Arabidopsis MTP members, they were named and their corresponding gene structure, chromosomal location, duplication events, and conserved motif were analyzed. The 12 CrMTPs were divided into 3 clusters (Zn-CDF, Fe/Zn-CDF, and Mn-CDF) according to their protein sequences, which had various structures and substrate specificities. The expression profiles of $\mathrm{CrMTP}$ genes based on transcriptome data were also analyzed in detail to explore the possible biological functions. The expression profiles of CrMTPs varied with different degrees among $C$. rosea tissues and in response to a series of HM treatments, which suggested that $C r M T P$ s performed a range of functions in specific tissues responding to environmental stress. Furthermore, RNA-seq data were used to verify whether CrMTPs played roles in the abiotic stress adaptation of different habitats. The functional identification of several CrMTP members in yeast confirmed substrate specificity for transporting transition metals. These results supply basic and important information for understanding the putative functions of CrMTPs in C. rosea involved in phytoremediation, which concerns HM pollution. Moreover, this is the first study to confirm that CrMTPs play possible roles in salt/alkaline tolerance, providing a basis for further understanding the role of $C r M T P$ family in C. rosea plants' adaptation to extreme adversity on tropical coral islands or reefs.

Supplementary Materials: The following are available online at https://www.mdpi.com/article/ 10.3390/plants10071340/s1, Figure S1: The molecular structures of CrMTPs, Figure S2: The FPKM values histogram of RNA-seq data in this study, Figure S3: Quantitative RT-PCR detection of the expression levels of $C r M T P s$ responding to different stresses (600 $\mathrm{mM} \mathrm{NaCl}$ and $100 \mathrm{mM} \mathrm{ABA}$ ) in C. rosea seedling plants, Figure S4: Simple map of CrMTPs-pYES2, Table S1: The sequences of CrMTP genomic DNA, CDS, and promoter region DNA, Table S2: Primer sequences used in this study.

Author Contributions: Conceptualization, M.Z. and S.J.; methodology, M.Z.; software, Z.W.; validation, Z.W. and Q.M.; formal analysis, T.Z.; investigation, R.L.; resources, R.L.; data curation, Z.W.; writing—original draft preparation, M.Z. and T.Z.; writing—-review and editing, M.Z.; visualization, T.Z. and L.P.; supervision, M.Z.; project administration, S.J.; funding acquisition, S.J. All authors have read and agreed to the published version of the manuscript.

Funding: This research was funded by the National Natural Sciences Foundation of China (31570257), the NSFC-Guangdong Province Union Funds (U1701246), the Guangdong Science and Technology Program (2019B121201005), and the Key Special Project for Introduced Talents Team of Southern Marine Science and Engineering Guangdong Laboratory (Guangzhou) (GML2019ZD0408). The funders had no roles in the study design, data collection, and analysis, decision to publish, or preparation of the manuscript.

Institutional Review Board Statement: Not applicable. 
Informed Consent Statement: Not applicable.

Data Availability Statement: Data are contained within the article.

Acknowledgments: The authors extend their appreciation to the workers who provided us Canavalia rosea plants in South China Botanical Garden (Guangzhou city, China) and C. rosea seeds gathered from Hainan Province, China.

Conflicts of Interest: The authors declare no conflict of interest.

\section{References}

1. Rascio, N.; Navari-Izzo, F. Heavy metal hyperaccumulating plants: How and why do they do it? and what makes them so interesting? Plant Sci. 2011, 180, 169-181. [CrossRef] [PubMed]

2. Clemens, S.; Ma, J.F. Toxic heavy metal and metalloid accumulation in crop plants and foods. Annu. Rev. Plant Biol. 2016, 67, 489-512. [CrossRef] [PubMed]

3. Menguer, P.K.; Farthing, E.; Peaston, K.A.; Ricachenevsky, F.K.; Fett, J.P.; Williams, L.E. Functional analysis of the rice vacuolar zinc transporter OsMTP1. J. Exp. Bot. 2013, 64, 2871-2883. [CrossRef] [PubMed]

4. Hall, J.L.; Williams, L.E. Transition metal transporters in plants. J. Exp. Bot. 2003, 54, 2601-2613. [CrossRef]

5. Montanini, B.; Blaudez, D.; Jeandroz, S.; Sanders, D.; Chalot, M. Phylogenetic and functional analysis of the cation diffusion facilitator (CDF) family: Improved signature and prediction of substrate specificity. BMC Genom. 2007, 8, 107. [CrossRef]

6. Nies, D.H.; Silver, S. Ion efflux systems involved in bacterial metal resistances. J. Ind. Microbiol. 1995, 14, 186-199. [CrossRef]

7. Gustin, J.L.; Zanis, M.J.; Salt, D.E. Structure and evolution of the plant cation diffusion facilitator family of ion transporters. BMC Evol. Biol. 2011, 11, 76. [CrossRef]

8. van der Zaal, B.J.; Neuteboom, L.W.; Pinas, J.E.; Chardonnens, A.N.; Schat, H.; Verkleij, J.A.; Hooykaas, P.J. Overexpression of a novel Arabidopsis gene related to putative zinc-transporter genes from animals can lead to enhanced zinc resistance and accumulation. Plant Physiol. 1999, 119, 1047-1055. [CrossRef]

9. Kobae, Y.; Uemura, T.; Sato, M.H.; Ohnishi, M.; Mimura, T.; Nakagawa, T.; Maeshima, M. Zinc transporter of Arabidopsis thaliana AtMTP1 is localized to vacuolar membranes and implicated in zinc homeostasis. Plant Cell Physiol. 2004, 45, 1749-1758. [CrossRef]

10. Blaudez, D.; Kohler, A.; Martin, F.; Sanders, D.; Chalot, M. Poplar metal tolerance protein 1 confers zinc tolerance and is an oligomeric vacuolar zinc transporter with an essential leucine zipper motif. Plant Cell 2003, 15, 2911-2928. [CrossRef]

11. Wang, F.H.; Qiao, K.; Liang, S.; Tian, S.Q.; Tian, Y.B.; Wang, H.; Chai, T.Y. Triticum urartu MTP1: Its ability to maintain Zn ${ }^{2+}$ and $\mathrm{Co}^{2+}$ homeostasis and metal selectivity determinants. Plant Cell Rep. 2018, 37, 1653-1666. [CrossRef]

12. Das, N.; Bhattacharya, S.; Maiti, M.K. Enhanced cadmium accumulation and tolerance in transgenic tobacco overexpressing rice metal tolerance protein gene OsMTP1 is promising for phytoremediation. Plant Physiol. Biochem. 2016, 105, 297-309. [CrossRef]

13. Barber-Zucker, S.; Shaanan, B.; Zarivach, R. Transition metal binding selectivity in proteins and its correlation with the phylogenomic classification of the cation diffusion facilitator protein family. Sci. Rep. 2017, 7, 16381. [CrossRef]

14. Fu, X.Z.; Tong, Y.H.; Zhou, X.; Ling, L.L.; Chun, C.P.; Cao, L.; Zeng, M.; Peng, L.Z. Genome-wide identification of sweet orange (Citrus sinensis) metal tolerance proteins and analysis of their expression patterns under zinc, manganese, copper, and cadmium toxicity. Gene 2017, 629, 1-8. [CrossRef]

15. Podar, D.; Scherer, J.; Noordally, Z.; Herzyk, P.; Nies, D.; Sanders, D. Metal selectivity determinants in a family of transition metal transporters. J. Biol. Chem. 2012, 287, 3185-3196. [CrossRef]

16. Arrivault, S.; Senger, T.; Krämer, U. The Arabidopsis metal tolerance protein AtMTP3 maintains metal homeostasis by mediating Zn exclusion from the shoot under Fe deficiency and Zn oversupply. Plant J. 2006, 46, 861-879. [CrossRef]

17. Peiter, E.; Montanini, B.; Gobert, A.; Pedas, P.; Husted, S.; Maathuis, F.J.; Blaudez, D.; Chalot, M.; Sanders, D. A secretory pathway-localized cation diffusion facilitator confers plant manganese tolerance. Proc. Natl. Acad. Sci. USA 2007, 104, 8532-8537. [CrossRef]

18. Delhaize, E.; Gruber, B.D.; Pittman, J.K.; White, R.G.; Leung, H.; Miao, Y.; Jiang, L.; Ryan, P.R.; Richardson, A.E. A role for the AtMTP11 gene of Arabidopsis in manganese transport and tolerance. Plant J. 2007, 51, 198-210. [CrossRef]

19. Fujiwara, T.; Kawachi, M.; Sato, Y.; Mori, H.; Kutsuna, N.; Hasezawa, S.; Maeshima, M. A high molecular mass zinc transporter MTP12 forms a functional heteromeric complex with MTP5 in the Golgi in Arabidopsis thaliana. FEBS J. 2015, 282, 1965-1979. [CrossRef]

20. Eroglu, S.; Meier, B.; von Wirén, N.; Peiter, E. The vacuolar manganese transporter MTP8 determines tolerance to iron deficiencyinduced chlorosis in Arabidopsis. Plant Physiol. 2016, 170, 1030-1045. [CrossRef]

21. Eroglu, S.; Giehl, R.F.H.; Meier, B.; Takahashi, M.; Terada, Y.; Ignatyev, K.; Andresen, E.; Küpper, H.; Peiter, E.; von Wirén, N. Metal tolerance protein 8 mediates manganese homeostasis and iron reallocation during seed development and germination. Plant Physiol. 2017, 174, 1633-1647. [CrossRef] [PubMed]

22. Chu, H.H.; Car, S.; Socha, A.L.; Hindt, M.N.; Punshon, T.; Guerinot, M.L. The Arabidopsis MTP8 transporter determines the localization of manganese and iron in seeds. Sci. Rep. 2017, 7, 11024. [CrossRef]

23. Yuan, L.; Yang, S.; Liu, B.; Zhang, M.; Wu, K. Molecular characterization of a rice metal tolerance protein, OsMTP1. Plant Cell Rep . 2012, 31, 67-79. [CrossRef] 
24. Chen, Z.; Fujii, Y.; Yamaji, N.; Masuda, S.; Takemoto, Y.; Kamiya, T.; Yusuyin, Y.; Iwasaki, K.; Kato, S.; Maeshima, M.; et al. Mn tolerance in rice is mediated by MTP8.1, a member of the cation diffusion facilitator family. J. Exp. Bot. 2013, 64, 4375-4387. [CrossRef]

25. Chen, X.; Li, J.; Wang, L.; Ma, G.; Zhang, W. A mutagenic study identifying critical residues for the structure and function of rice manganese transporter OsMTP8.1. Sci. Rep. 2016, 6, 32073. [CrossRef]

26. Takemoto, Y.; Tsunemitsu, Y.; Fujii-Kashino, M.; Mitani-Ueno, N.; Yamaji, N.; Ma, J.F.; Kato, S.I.; Iwasaki, K.; Ueno, D. The tonoplast-localized transporter MTP8.2 contributes to manganese detoxification in the shoots and roots of Oryza sativa L. Plant Cell Physiol. 2017, 58, 1573-1582. [CrossRef]

27. Tsunemitsu, Y.; Yamaji, N.; Ma, J.F.; Kato, S.I.; Iwasaki, K.; Ueno, D. Rice reduces Mn uptake in response to Mn stress. Plant Signal. Behav. 2018, 13, e1422466. [CrossRef]

28. Zhang, M.; Liu, B. Identification of a rice metal tolerance protein OsMTP11 as a manganese transporter. PLoS ONE 2017, 12, e0174987. [CrossRef]

29. Farthing, E.C.; Menguer, P.K.; Fett, J.P.; Williams, L.E. OsMTP11 is localized at the Golgi and contributes to Mn tolerance. Sci. Rep. 2017, 7, 15258. [CrossRef]

30. Ma, G.; Li, J.; Li, J.; Li, Y.; Gu, D.; Chen, C.; Cui, J.; Chen, X.; Zhang, W. OsMTP11, a trans-Golgi network localized transporter, is involved in manganese tolerance in rice. Plant Sci. 2018, 274, 59-69. [CrossRef]

31. Migocka, M.; Małas, K.; Maciaszczyk-Dziubinska, E.; Posyniak, E.; Migdal, I.; Szczech, P. Cucumber Golgi protein CsMTP5 forms a Zn-transporting heterodimer with high molecular mass protein CsMTP12. Plant Sci. 2018, 277, 196-206. [CrossRef] [PubMed]

32. Persans, M.W.; Nieman, K.; Salt, D.E. Functional activity and role of cation-efflux family members in Ni hyperaccumulation in Thlaspi goesingense. Proc. Natl. Acad. Sci. USA 2001, 98, 9995-10000. [CrossRef] [PubMed]

33. Kim, D.; Gustin, J.L.; Lahner, B.; Persans, M.W.; Baek, D.; Yun, D.J.; Salt, D.E. The plant CDF family member TgMTP1 from the $\mathrm{Ni} / \mathrm{Zn}$ hyperaccumulator Thlaspi goesingense acts to enhance efflux of $\mathrm{Zn}$ at the plasma membrane when expressed in Saccharomyces cerevisiae. Plant J. 2004, 39, 237-251. [CrossRef] [PubMed]

34. Gustin, J.L.; Loureiro, M.E.; Kim, D.; Na, G.; Tikhonova, M.; Salt, D.E. MTP1-dependent Zn sequestration into shoot vacuoles suggests dual roles in Zn tolerance and accumulation in Zn-hyperaccumulating plants. Plant J. 2009, 57, 1116-1127. [CrossRef]

35. Dräger, D.B.; Desbrosses-Fonrouge, A.G.; Krach, C.; Chardonnens, A.N.; Meyer, R.C.; Saumitou-Laprade, P.; Krämer, U. Two genes encoding Arabidopsis halleri MTP1 metal transport proteins co-segregate with zinc tolerance and account for high MTP1 transcript levels. Plant J. 2004, 39, 425-439. [CrossRef]

36. Shahzad, Z.; Gosti, F.; Frérot, H.; Lacombe, E.; Roosens, N.; Saumitou-Laprade, P.; Berthomieu, P. The five AhMTP1 zinc transporters undergo different evolutionary fates towards adaptive evolution to zinc tolerance in Arabidopsis halleri. PLoS Genet. 2010, 6, e1000911. [CrossRef]

37. Delhaize, E.; Kataoka, T.; Hebb, D.M.; White, R.G.; Ryan, P.R. Genes encoding proteins of the cation diffusion facilitator family that confer manganese tolerance. Plant Cell 2003, 15, 1131-1142. [CrossRef]

38. Zhang, M.; Senoura, T.; Yang, X.; Nishizawa, N.K. Functional analysis of metal tolerance proteins isolated from Zn/Cd hyperaccumulating ecotype and non-hyperaccumulating ecotype of Sedum alfredii Hance. FEBS Lett. 2011, 585, 2604-2609. [CrossRef]

39. Xu, J.; Chai, T.; Zhang, Y.; Lang, M.; Han, L. The cation-efflux transporter BjCET2 mediates zinc and cadmium accumulation in Brassica juncea L. leaves. Plant Cell Rep. 2009, 28, 1235-1242. [CrossRef]

40. Lang, M.; Hao, M.; Fan, Q.; Wang, W.; Mo, S.; Zhao, W.; Zhou, J. Functional characterization of BjCET3 and BjCET4, two new cation-efflux transporters from Brassica juncea L. J. Exp. Bot. 2011, 62, 4467-4480. [CrossRef]

41. Nikalje, G.C.; Suprasanna, P. Coping with metal toxicity-cues from halophytes. Front. Plant Sci. 2018, 9, 777. [CrossRef]

42. Lutts, S.; Lefèvre, I. How can we take advantage of halophyte properties to cope with heavy metal toxicity in salt-affected areas? Ann. Bot. 2015, 115, 509-528. [CrossRef]

43. Khalilzadeh, R.; Pirzad, A.; Sepehr, E.; Khan, S.; Anwar, S. The Salicornia europaea potential for phytoremediation of heavy metals in the soils under different times of wastewater irrigation in northwestern Iran. Environ. Sci. Pollut. Res. Int. 2021, 1-14. [CrossRef]

44. Alam, M.R.; Islam, R.; Anh Tran, T.K.; Van, D.L.; Rahman, M.M.; Griffin, A.S.; Yu, R.M.K.; MacFarlane, G.R. Global patterns of accumulation and partitioning of metals in halophytic saltmarsh taxa: A phylogenetic comparative approach. J. Hazard Mater. 2021, 414, 125515. [CrossRef]

45. Shang, C.; Wang, L.; Tian, C.; Song, J. Heavy metal tolerance and potential for remediation of heavy metal-contaminated saline soils for the euhalophyte Suaeda salsa. Plant Signal. Behav. 2020, 15, 1805902. [CrossRef]

46. Kulkarni, R.; Deobagkar, D.; Zinjarde, S. Metals in mangrove ecosystems and associated biota: A global perspective. Ecotoxicol. Environ. Saf. 2018, 153, 215-228. [CrossRef]

47. Pittarello, M.; Busato, J.G.; Carletti, P.; Dobbss, L.B. Possible developments for ex situ phytoremediation of contaminated sediments, in tropical and subtropical regions-Review. Chemosphere 2017, 182, 707-719. [CrossRef]

48. Nosek, M.; Kaczmarczyk, A.; Jędrzejczyk, R.J.; Supel, P.; Kaszycki, P.; Miszalski, Z. Expression of genes involved in heavy metal trafficking in plants exposed to salinity stress and elevated Cd concentrations. Plants 2020, 9, 475. [CrossRef]

49. Yin, D.L.; Peng, F.C.; He, T.R.; Xu, Y.Y.; Wang, Y.M. Ecological risks of heavy metals as influenced by water-level fluctuations in a polluted plateau wetland, southwest China. Sci. Total Environ. 2020, 742, 140319. [CrossRef] 
50. Tanaka, N.; Fujiwara, T.; Tomioka, R.; Krämer, U.; Kawachi, M.; Maeshima, M. Characterization of the histidine-rich loop of Arabidopsis vacuolar membrane zinc transporter AtMTP1 as a sensor of zinc level in the cytosol. Plant Cell Physiol. 2015, 56, 510-519. [CrossRef]

51. Gao, Y.; Yang, F.; Liu, J.; Xie, W.; Zhang, L.; Chen, Z.; Peng, Z.; Ou, Y.; Yao, Y. Genome-wide identification of metal tolerance protein genes in Populus trichocarpa and their roles in response to various heavy metal stresses. Int. J. Mol. Sci. 2020, 21, 1680. [CrossRef]

52. Liu, J.; Gao, Y.; Tang, Y.; Wang, D.; Chen, X.; Yao, Y.; Guo, Y. Genome-wide identification, comprehensive gene feature, evolution, and expression analysis of plant metal tolerance proteins in tobacco under heavy metal toxicity. Front. Genet. 2019, 10, 345. [CrossRef]

53. Zhang, X.; Li, Q.; Xu, W.; Zhao, H.; Guo, F.; Wang, P.; Wang, Y.; Ni, D.; Wang, M.; Wei, C. Identification of MTP gene family in tea plant (Camellia sinensis L.) and characterization of CsMTP8.2 in manganese toxicity. Ecotoxicol. Environ. Saf. 2020, 202, 110904. [CrossRef]

54. Li, X.; Wu, Y.; Li, B.; He, W.; Yang, Y.; Yang, Y. Genome-wide identification and expression analysis of the cation diffusion facilitator gene family in turnip under diverse metal ion stresses. Front. Genet. 2018, 9, 103. [CrossRef]

55. Kolaj-Robin, O.; Russell, D.; Hayes, K.A.; Pembroke, J.T.; Soulimane, T. Cation diffusion facilitator family: Structure and function. FEBS Lett. 2015, 589, 1283-1295. [CrossRef]

56. Vatansever, R.; Filiz, E.; Eroglu, S. Genome-wide exploration of metal tolerance protein (MTP) genes in common wheat (Triticum aestivum): Insights into metal homeostasis and biofortification. Biometals 2017, 30, 217-235. [CrossRef]

57. Shirazi, Z.; Abedi, A.; Kordrostami, M.; Burritt, D.J.; Hossain, M.A. Genome-wide identification and characterization of the metal tolerance protein (MTP) family in grape (Vitis vinifera L.). 3 Biotech 2019, 9, 199. [CrossRef]

58. Ray, R.; Mandal, S.K.; González, A.G.; Pokrovsky, O.S.; Jana, T.K. Storage and recycling of major and trace element in mangroves. Sci. Total Environ. 2021, 780, 146379. [CrossRef]

59. Wittkopp, P.J.; Kalay, G. Cis-regulatory elements: Molecular mechanisms and evolutionary processes underlying divergence. Nat. Rev. Genet. 2011, 13, 59-69. [CrossRef]

60. Zhang, W.; Liao, X.; Cui, Y.; Ma, W.; Zhang, X.; Du, H.; Ma, Y.; Ning, L.; Wang, H.; Huang, F.; et al. A cation diffusion facilitator, GmCDF1, negatively regulates salt tolerance in soybean. PLoS Genet. 2019, 15, e1007798. [CrossRef]

61. Xu, T.; Chen, H.; Li, J.; Hong, S.; Shao, L.; Zheng, X.; Zou, Q.; Wang, Y.; Guo, S.; Jiang, J. Implications for cation selectivity and evolution by a novel cation diffusion facilitator family member from the moderate halophile Planococcus dechangensis. Front. Microbiol. 2019, 10, 607. [CrossRef]

62. Krämer, U. MTP1 mops up excess zinc in Arabidopsis cells. Trends Plant Sci. 2005, 10, 313-315. [CrossRef] [PubMed]

63. Mulder, N.; Apweiler, R. InterPro and InterProScan: Tools for protein sequence classification and comparison. Methods Mol. Biol. 2007, 396, 59-70. [CrossRef] [PubMed]

64. Mistry, J.; Chuguransky, S.; Williams, L.; Qureshi, M.; Salazar, G.A.; Sonnhammer, E.L.L.; Tosatto, S.C.E.; Paladin, L.; Raj, S.; Richardson, L.J.; et al. Pfam: The protein families database in 2021. Nucleic Acids Res. 2021, 49, D412-D419. [CrossRef]

65. Buchfink, B.; Xie, C.; Huson, D.H. Fast and sensitive protein alignment using DIAMOND. Nat. Methods 2015, 12, 59-60. [CrossRef] [PubMed]

66. Nei, M.; Gojobori, T. Simple methods for estimating the numbers of synonymous and nonsynonymous nucleotide substitutions. Mol. Biol. Evol. 1986, 3, 418-426. [CrossRef] [PubMed]

67. Chen, C.; Chen, H.; Zhang, Y.; Thomas, H.R.; Frank, M.H.; He, Y.; Xia, R. TBtools: An integrative toolkit developed for interactive analyses of big biological data. Mol. Plant 2020, 13, 1194-1202. [CrossRef] [PubMed]

68. Searle, P.F.; Stuart, G.W.; Palmiter, R.D. Metal regulatory elements of the mouse metallothionein-I gene. Exp. Suppl. 1987, 52, 407-414. [CrossRef]

69. Wimalarathna, R.N.; Pan, P.Y.; Shen, C.H. Chromatin repositioning activity and transcription machinery are both recruited by Ace1p in yeast CUP1 activation. Biochem. Biophys. Res. Commun. 2012, 422, 658-663. [CrossRef]

70. Quinn, J.M.; Merchant, S. Two copper-responsive elements associated with the Chlamydomonas Cyc6 gene function as targets for transcriptional activators. Plant Cell 1995, 7, 623-628. [CrossRef]

71. Gietz, R.D. Yeast transformation by the LiAc/SS carrier DNA/PEG method. Methods Mol. Biol. 2014, 1205, 1-12. [CrossRef] 